Maestría en Procesamiento de SeÑAles e IMÁgenes Digitales

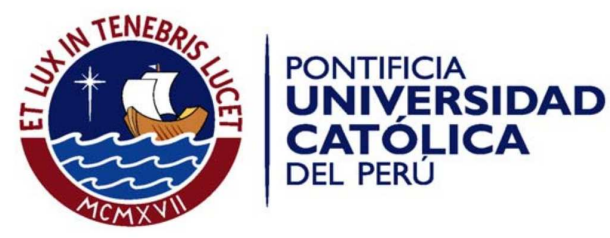

ESCUELA DE POSGRADO

PONTIFICIA UNIVERSIDAD CATÓLICA DEL PERÚ

\title{
Regularized Spectral Log Difference Technique for Ultrasonic Attenuation Imaging
}

\author{
Submitted by
}

\section{Andres Leonel Coila Pacompia}

\author{
In partial fulfillment of the requirements for the Degree of \\ Thesis supervised by: \\ Roberto Lavarello \\ Examining commitee members: \\ Roberto Lavarello \\ Michael L. Oelze \\ Tomy Varghese \\ Lima, Perú \\ June 9, 2017
} Master in Digital Signal and Image Processing in the Graduate School of the Pontificia Universidad Católica del Perú. 


\begin{abstract}
The attenuation coefficient slope (ACS) has the potential to be used for tissue characterization and as a diagnostic ultrasound tool, hence complementing B-mode images. The ACS can be valuable for estimation of other ultrasound parameters such as the backscatter coefficient. There is a well-known tradeoff between the precision of the estimated ACS values and the data block size used in spectral-based techniques such as the spectral log difference (SLD). This trade-off limits the practical usefulness of spectral-based attenuation imaging techniques.

In this thesis work, the regularized spectral log difference (RSLD) technique is presented in detail and evaluated with simulations and experiments with physical phantoms, ex vivo and in vivo. The ACS values obtained when using the RSLD technique were compared to the ones obtained when using the SLD technique, as well as the ground truth ACS values obtained with insertion loss techniques. The results showed that the RSLD technique allowed significantly decreasing estimation variance when using small data block sizes (i.e., standard deviation of percentage error reduced by more than an order of magnitude in all cases when using $10 \lambda \times 10 \lambda$ data blocks) without sacrificing estimation accuracy. Therefore, the RSLD allows for the reconstruction of attenuation coefficient images with an improved trade-off between spatial resolution and estimation precision.
\end{abstract}


To .

My mother Mercedes, my father Andres and my sisters Nury, Gladys, Mabel and Flor. 


\section{Acknowledgments}

This thesis work closes my master studies chapter. I want to thank my family for their help as they always supported me in the way I most needed, and without any doubt it was due to their help that I could finish this work. I want to acknowledge Profs. Roberto Lavarello and Benjamin Castaneda for welcoming me into the Laboratorio de Imagenes Medicas (LIM) and always providing useful comments. Thanks to LIM members that helped me since the beginning and also thanks to my friends in the M.Sc. program for sharing many amusing and challenging moments with me. I would also like to thank several people that in one way or another helped me improving personally and professionally these last two years both inside and outside PUCP. Finally, thanks to my funding sources: Fondo Nacional de Desarrollo Cientifico y Tecnologico-PERU under Grant 012-2014-FONDECYT-C1 from the Peruvian Government, and the Direccion de Gestion de la Investigacion DGI-PUCP through grant PAIP-2016. 


\section{Contents}

1 Introduction $\quad 1$

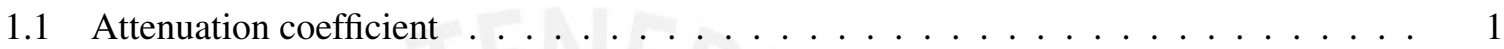

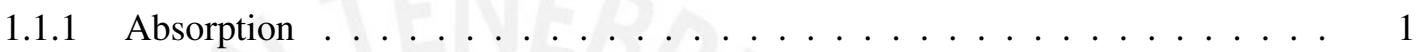

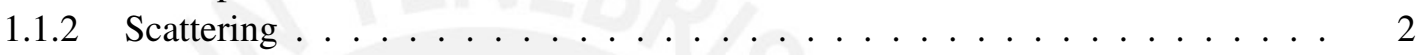

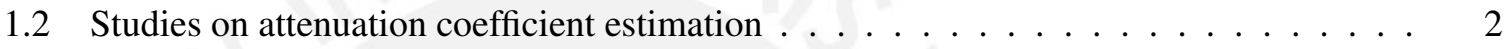

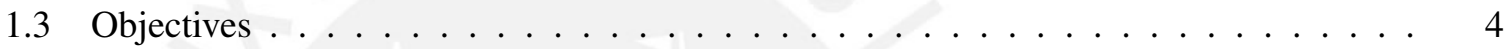

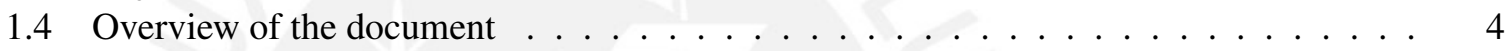

2 Attenuation estimation methods $\quad 6$

2.1 Spectral $\log$ difference technique . . . . . . . . . . . . . . . . 6

2.2 Diffraction compensation functions for the spectral $\log$ difference $\ldots \ldots \ldots$

2.2.1 Spherically focused transducers . . . . . . . . . . . . . . 8

2.2 .2 Linear array transducers . . . . . . . . . . . . . . . . . . . 8

2.3 Regularized spectral $\log$ difference technique $\ldots \ldots \ldots \ldots \ldots$

2.3 .1 RSLD derivation . . . . . . . . . . . . . . . . . . . . 9

2.3.2 RSLD implementation . . . . . . . . . . . . . . . . . 10

3 Simulations and experiments $\quad 11$

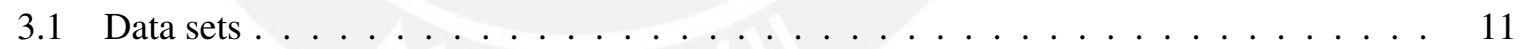

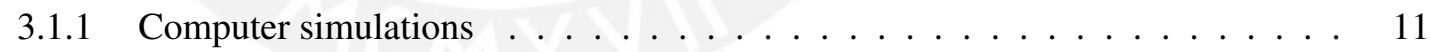

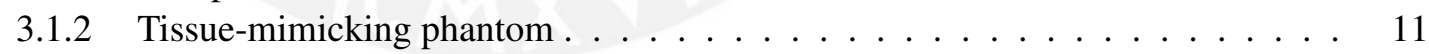

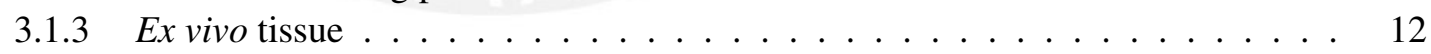

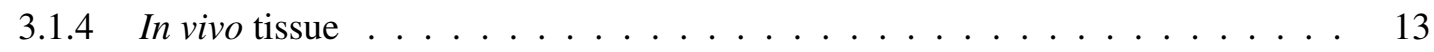

3.2 Construction of attenuation coefficient maps . . . . . . . . . . . . . . . 14

3.3 Evaluation of attenuation coefficient maps . . . . . . . . . . . . . . . . . 14

3.3.1 Mean and standard deviation . . . . . . . . . . . . . . . . . . . . . . . . . . . . . . . . . 14

3.3 .2 Percentage error . . . . . . . . . . . . . . . . . . . . 14

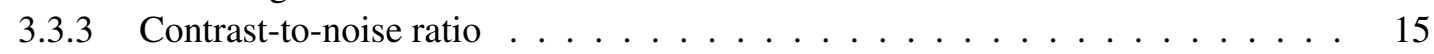

4 Testing and Validation 16

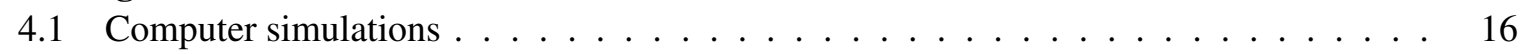

4.1 .1 Homogeneous simulation $\ldots \ldots \ldots \ldots \ldots$

4.1 .2 Inhomogeneous simulation . . . . . . . . . . . . . . . 16

4.2 Tissue-mimicking phantom . . . . . . . . . . . . . . . . . . . 19

4.2 .1 Physical phantom $\# 1 \ldots \ldots \ldots \ldots$ 
4.2.2 Physical phantom $\# 2 \ldots \ldots \ldots$

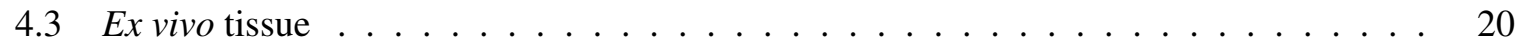

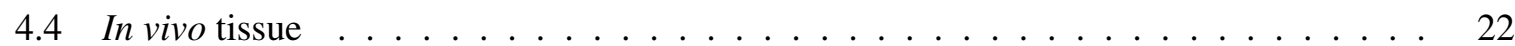

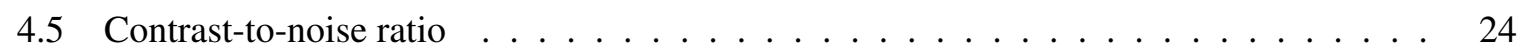

4.6 Impact of the regularization parameter value . . . . . . . . . . . 25

5 Conclusion $\quad 26$

$\begin{array}{ll}\text { Bibliography } & 27\end{array}$ 


\section{List of Figures}

2.1 Data block beginning at depth $z_{0}$, in dashed red are depicted both proximal (center at $z_{p}$ depth) and distal (center at $z_{d}$ depth) windows. . . . . . . . . . . . . 7

2.2 Spectral $\log$ ratio vs. frequency. . . . . . . . . . . . . . . 7

2.3 Measured log ratios and attenuation coefficient slope $\beta$ corresponding to the data blocks within the total field of view allocated in a 2 D grid. . . . . . . . . . . . . . . .

3.1 Inhomogeneous attenuation coefficient map in $\mathrm{dB} \cdot \mathrm{cm}^{-1} \cdot \mathrm{MHz}^{-1}$ used for simulating radiofrequency backscattered echoes. . . . . . . . . . . . . . . . 12

3.2 B-mode images of Phantom \#1 (a) and Phantom \#2 (b) . . . . . . . . . . . . . . 12

3.3 Procedure for the data acquistion from the ex vivo chicken breast tissue embedded into

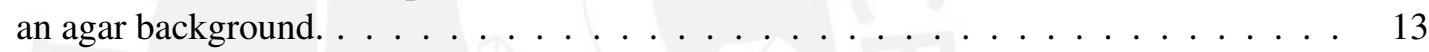

3.4 B-mode image of in vivo breast tumor. . . . . . . . . . . . . . . . . . 13

4.1 B-mode image for Simulation \#1 (a) and ACS maps when using the SLD (b)-(d) and RSLD (e)-(g) techniques for data block sizes of $50 \lambda \times 50 \lambda, 30 \lambda \times 30 \lambda$ and $20 \lambda \times 20 \lambda$, respectively. . . . . . . . . . . . . . . . . . .

4.2 B-mode image for Simulation \#2 (a) and ACS maps when using the SLD (b)-(d) and RSLD (e)-(g) techniques, respectively, for data block sizes of $40 \lambda \times 40 \lambda, 30 \lambda \times 30 \lambda$ and $20 \lambda \times 20 \lambda$. The dashed and solid lines outline the inclusion and regions for the calculation of performance metrics, respectively. . . . . . . . . . . . . . .

4.3 B-mode image for Phantom\#1 (a) and ACS maps when using the SLD (b)-(d) and RSLD (e)-(g) techniques, respectively, for data block sizes of $40 \lambda \times 40 \lambda, 30 \lambda \times 30 \lambda$ and $20 \lambda \times$ $20 \lambda$. The dashed and solid lines outline the inclusion and regions for the calculation of performance metrics, respectively. . . . . . . . . . . . . . .

4.4 B-mode image for Phantom\#2 (a) and ACS maps when using the SLD (b)-(d) and RSLD (e)-(g) techniques, respectively, for data block sizes of $40 \lambda \times 40 \lambda, 30 \lambda \times 30 \lambda$ and $20 \lambda \times$ $20 \lambda$. The dashed and solid lines outline the inclusion and regions for the calculation of performance metrics, respectively. . . . . . . . . . . . . . . .

4.5 B-mode image for ex vivo chicken breast tissue (a) and ACS maps when using the SLD (b)-(d) and RSLD (e)-(g) techniques for data block sizes of $40 \lambda \times 40 \lambda, 30 \lambda \times 30 \lambda$ and $20 \lambda \times 20 \lambda$, respectively. The arrows and solid lines outline the inclusion and regions for the calculation of performance metrics, respectively. . . . . . . . . . . . . .

4.6 B-mode image for in vivo breast tissue (a) and ACS maps when using the SLD (b)(d) and RSLD (e)-(g) techniques for data block sizes of $30 \lambda \times 30 \lambda, 20 \lambda \times 20 \lambda$ and $10 \lambda \times 10 \lambda$, respectively. The arrows and solid lines outline the tumor and regions for the calculation of performance metrics, respectively. . . . . . . . . . . . 
4.7 CNR of the SLD (a), RSLD (b) and the gain in CNR of the RSLD with respect to the SLD technique (c) of ACS maps for different data block sizes. . . . . . . . . . . . . .

4.8 Effects of the regularization parameter in the reconstruction of ACS maps using the Phantom \#1. (a) Mean and standard deviation of the inclusion (square markers) and background (diamond markers). The horizontal lines indicate the ground truth values for each region. (b) CNR. (c) MPE of the inclusion (square markers) and background (diamond markers). The horizontal dashed lines mark 10\% and $20 \%$ bias levels. . . . . 


\section{List of Tables}

3.1 Frequency range and wavelength size of the data sets used in this work for testing the SLD and RSLD techniques. . . . . . . . . . . . . . . . . . . . . .

4.1 Estimated ACS mean, standard deviation, MPE and SDPE for Simulation \#1 obtained when using the SLD and the RSLD techniques. The ground truth ACS value was 0.50 $\mathrm{db} \cdot \mathrm{cm}^{-1} \cdot \mathrm{MHz}^{-1}$.

4.2 Estimated ACS mean, standard deviation, MPE and SDPE for Simulation \#2 obtained when using the SLD and the RSLD techniques. The ground truth ACS value was 1.0 $\mathrm{db} . \mathrm{cm}^{-1} \cdot \mathrm{MHz}^{-1} \ldots \ldots \ldots \ldots \ldots \ldots \ldots$

4.3 Estimated ACS mean, standard deviation, MPE and SDPE of inclusion for Phantom \#1 obtained when using the SLD and the RSLD techniques. The ground truth ACS value was $1.04 \mathrm{db} . \mathrm{cm}^{-1} . \mathrm{MHz}^{-1}$.

4.4 Estimated ACS mean, standard deviation, MPE and SDPE of inclusion for Phantom \#2 obtained when using the SLD and the RSLD techniques. The ground truth ACS value

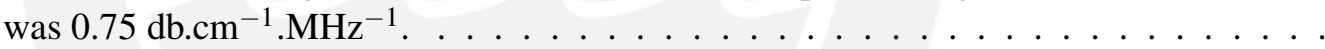

4.5 Estimated ACS mean, standard deviation, MPE and SDPE of ex vivo chicken breast tissue obtained when using the SLD and the RSLD techniques. The ground truth ACS value was $1.24 \mathrm{db} . \mathrm{cm}^{-1} . \mathrm{MHz}^{-1}$.

4.6 Estimated ACS mean and standard deviation of tumor and normal tissue for in vivo tissue obtained when using the SLD and the RSLD techniques. . . . . . . . . . . . . . 


\section{Chapter 1}

\section{Introduction}

Ultrasound are mechanical waves with frequencies $(f)$ above the human hearing limit (i.e., $f>20$ $\mathrm{KHz}$ ). Ultrasound has been applied extensively in last decades for medical tasks including the use of pulse-echo techniques for imaging human body internal structures [1]. Quantitative ultrasound (QUS) encompasses a set of techniques designed to provide numerical values of intrinsic tissue properties [2]. The attenuation coefficient is one of the QUS parameters widely explored for tissue characterization purposes.

\subsection{Attenuation coefficient}

Ultrasonic attenuation is the progressive loss of ultrasound wave energy as the ultrasound passess through a medium (e.g., human tissues). Attenuation is modeled as an exponential function of propagation depth. For a 1-D ultrasound wave the pressure $p(\mathrm{x}, \mathrm{t})$ as a function of the time $(\mathrm{t})$ and distance (x) can be expressed as

$$
p(\mathrm{x}, \mathrm{t})=A_{0} e^{-\alpha \mathrm{x}} e^{j\left(\omega \mathrm{t}-k_{r} \mathrm{x}\right)},
$$

where $e^{-\alpha \mathrm{x}}$ act as an exponential damping term. Moreover, high frequencies of the ultrasound wave are more attenuated than the low frequencies. This observation leads to the following expression for the ultrasonic attenuation $(\alpha)$ as a function of frequency

$$
\alpha(f)=\beta \times f^{\gamma},
$$

where $\beta$ and $\gamma$ are experimentally estimated from a fit of the ultrasonic attenuation function dependence of frequency $(f)$. For several soft tissues, the power law exponent $\gamma=1$ is a good approximation [1], although some tissues might have larger values (e.g. $\gamma_{\text {spleen }}=1.3$ and $\gamma_{\text {breast }}=1.5$ ). For $\gamma=1$, the $\beta$ value is named attenuation coefficient slope.

The ultrasonic attenuation is produced by several types of interactions between the ultrasound wave and the medium, being the most important ones the absorption and scattering [3, 4].

\subsubsection{Absorption}

The mechanisms of ultrasound absorption can be grouped in two categories: classical absorption and relaxational absorption. The total absorption coefficient depends on both mechanisms, being the latter the dominant one. 
Classical absorption refers to losses produced by the viscosity and thermal conductivity as the ultrasound wave passes across a medium. First, ultrasound wave energy losses are caused by displacements of particles. Second, losses by thermal conductivity occur when the heat flows from high temperature regions (i.e., compression regions) to low temperature regions (i.e., rarefaction regions). The classical mechanisms are typically dependent on $f^{2}$. On the other hand, relaxational absorption losses occur as the ultrasound energy is used for returning a distorted medium to the equilibrium. A time constant $\tau$ is associated with the absorption mechanisms.

\subsubsection{Scattering}

Scattering is produced when the ultrasound wave encounters structures in the tissue. The type of ultrasound scattering depend on the scatterer size (e.g. radius $=$ a) with respect to the ultrasound wavelength ( $\lambda$ ) defined as

$$
\lambda=\frac{\mathrm{c}_{s}}{f}
$$

where $\mathrm{c}_{s}$ is sound velocity in the medium and $f$ is the frequency of the ultrasound wave. The ultrasound scattering can be [1]

1. Specular scattering, if $\mathrm{a} \gg \lambda$. The backscattered intensity does not change with the frequency.

2. Diffusive or Rayleigh scattering, if a $\ll \lambda$. In this case, the ultrasound scattering occurs in all directions and the scattered intensity is proportional to the frequency to the fourth power and to the sixth power of the radius.

3. Diffractive scattering, if $\mathrm{a} \approx \lambda$.

The backscatter coefficient (BSC) is a property that quantifies the amount of energy that is reflected by a material as function of the frequency of the ultrasound wave. Therefore, the backscatter coefficient estimation habe been found useful for derive parameters of such material like the effective scatterer size and concentration.

\subsection{Studies on attenuation coefficient estimation}

Several studies have performed attenuation estimation in liver $[5,6,7,8,9]$, thyroid [10, 11], breast $[12,13,14]$, spleen [15], muscle [16], skin [17, 18], cervical and uterine tissues [19, 20] and small plaques [21]. A related parameter is the total attenuation that an ultrasonic wave experiences from the surface of the transducer to a region of analysis within a tissue of interest. The total attenuation along the ultrasound path is required for the estimation of other QUS parameters such as the backscatter coefficient [22] that might provide information of the effective size and density of scatterers in a region of interest. The estimation of the total ultrasound attenuation has been explored using several methods [23, 24], including an estimation derived directly from local attenuation coefficient maps [25].

The attenuation coefficient slope (ACS) can be estimated using techniques based on the spectral information from the radiofrequency (RF) backscattered echoes analyzing either the amplitude change (spectral difference, spectral log difference) or the downshift of the spectrum (centroid downshift, hybrid method) as a function of depth [26, 27, 28]. Samimi and Varghese [29] experimentally studied the achievable variance of the spectral difference technique, and found that data block sizes of 30 lateral lines by 10.5 axial wavelengths $(\lambda)$ and 10 lateral lines by 72 axial wavelengths resulted in standard 
deviations errors of approximately $70 \%$ and $20 \%$, respectively. Labyed and Bigelow [27] showed with simulations that when using the spectral log difference technique, standard deviations lower than $20 \%$ can be achieved when using data block sizes of 5 lateral lines by 70 axial wavelengths ( 28 pulse lengths) or 40 lateral lines by 26.25 axial wavelengths (10.5 pulse lengths). Therefore, the spatial resolution of the ACS estimation methods based on depth-dependent spectral amplitude changes is severely limited for practical applications.

The severe trade-off between spatial resolution and precision in ACS estimation also plagues methods that derive estimates from spectral downshifts. Samimi and Varghese [26] studied the performance of the centroid downshift technique, and reported that estimates from a physical phantom with ACS of $0.5 \mathrm{~dB} \cdot \mathrm{cm}^{-1} \cdot \mathrm{MHz}^{-1}$ resulted in standard deviations of $270 \%$ and $90 \%$ when using data blocks of 40 lines laterally by 18.5 and 30 wavelengths axially, respectively. Labyed and Bigelow [27] reported that the precision of the hybrid method was comparable to the one obtained with the spectral log difference technique, and therefore data blocks of several tens of wavelengths axially and laterally are required for obtaining precise estimates. In another study, Samimi and Varghese [30] developed an optimum frequency-shift estimator that enhances the hybrid method technique achieving for a physical phantom with ACS of $0.8 \mathrm{~dB} . \mathrm{cm}^{-1} \cdot \mathrm{MHz}^{-1}$ standard deviations of approximately $58.8 \%$ and $7.5 \%$ when using data block sizes of 40 lateral lines by 38 and 140 axial wavelengths, respectively. Therefore, research is still required to develop ACS estimators with a better trade-off between spatial resolution and precision.

All the results discussed so far were obtained with homogeneous phantoms either in simulations or experiments. Some results are also available when the assumptions required for deriving spectralbased ACS estimation methods break down. For example, Labyed and Bigelow [27] evaluated the spectral log difference technique with inhomogeneous simulations of data blocks with varying scatterer number density in the axial dimension as a step function. It was reported that standard deviation errors are higher than $25 \%$ even though using using data blocks with sizes of 5 lateral lines by 87.5 axial wavelengths (35 pulse lengths) or 100 lateral lines by 26.25 axial wavelengths (10.5 pulse lengths). Though, the hybrid method results were comparable to the spectral log difference technique, the spectral difference in the aforementioned inhomogeneous medium fails resoundingly, as the accuracy error was higher than $100 \%$. Therefore, it is evident the lack of a more robust method for ACS estimation in inhomogeneous media. In other study, Rubert and Varghese demonstrated that ACS precision with the spectral difference technique deteriorates rapidly when speckle deviates from Rayleigh statistics [31]. In particular, the standard deviation of ACS estimates increased from $11.5 \%$ to $51 \%$ when the envelope signal-to-noise ratio (SNR) decreases from 1.78 to 1.01 with a Siemens S2000 scanner equipped with a 9L4 array (nominal center frequency of $6.5 \mathrm{MHz}$ ). The data block size was $16 \mathrm{~mm}$ axially by $15 \mathrm{~mm}$ laterally, which roughly corresponds to $67 \lambda$ axially by $62 \lambda$ laterally. These results suggest that the performance of available spectral-based ACS estimation techniques is expected to be worse in clinical applications where simplified assumptions are not expected to hold.

Some spectral-based ACS estimation techniques have been used in tandem with compounding approaches. Tu et al. [32] used the spectral difference technique in a uniform physical phantom of 0.5 $\mathrm{dB} . \mathrm{cm}^{-1} \cdot \mathrm{MHz}^{-1}$ using data blocks of approximately $23 \lambda$ axially by $23 \lambda$ laterally along with spatial compounding and frequency compounding, respectively. It was reported that spatial compounding by averaging ACS values of 15 angles with $3.75^{\circ}$ steps decreased the standard deviation error from $84 \%$ to $24 \%$, whereas frequency compounding of ACS values resulted after applying the spectral difference technique at five frequencies resulted in standard deviation error decrease of $84 \%$ to $40 \%$. Whereas the compounding approaches looks promising, the need of multiple acquisitions for spatial compounding remains the most significant downside of this technique. In other study, Zenteno et al. [33] used a full 
angular spatial compounding (FASC) method by acquiring backscattered data of 30 angles (ranging from $0^{\circ}$ to $360^{\circ}$ ) with data block sizes of $29 \lambda$ axially by $29 \lambda$ laterally. The FASC technique application on physical phantoms resulted in variance reduction rates higher than $88 \%$ while holding the bias below $8 \%$. Unfortunately, the soft tissues where a full angular view can be achievable is limited mainly to breast.

Some recent efforts towards developing improved ACS estimators in the time-domain can be found in the literature. For example, Ghoshal and Oelze [34] enhance time-domain ACS estimation methods by coupling diffraction effects and attenuation effects. It was reported in physical phantoms a standard deviation error lower than $20 \%$ and $10 \%$ for a data block size of $22.5 \lambda$ axially by $43.8 \lambda$ laterally and $45 \lambda$ axially by $43.8 \lambda$ laterally, respectively. However, more studies are required to assess the performance. Other approaches such as the iterative reconstruction method proposed by Ilyina et al. [35] aims to derive ultrasound parameters such as attenuation, nonlinear distortion, reflection and scattering by minimizing the actual measured power spectra and the simulated power spectra that is modified iteratively by varying the aforementioned ultrasound parameters. In spite of having mainly ACS estimation bias less than $10 \%$, there is no information of variance. In addition, the assumption of a plane wave propagation model may be limited in clinical applications where diffraction effects of transducers need to be taken into account.

\subsection{Objectives}

Currently, spectral-based techniques construct attenuation coefficient maps by estimating ACS values from each data block at a time, therefore, requiring large sizes of data block to achieve reasonable values of variance as detailed in the literature. Estimating ACS values from each data block at a time does not exploit the two-dimensional nature of attenuation coefficient maps. Therefore, the present work aims to extend the trade-off between the data block size and the precision when reconstructing attenuation coefficients. As a result, relatively small data block sizes (i.e., $20 \lambda$ axially by $20 \lambda$ laterally or less) can be used while obtaining attenuation coefficient maps with typical coefficients of variation of less than $25 \%$. The specific objectives of this work are:

1. Develop a joint attenuation coefficient estimator that exploits the two dimensional nature of the attenuation coefficient maps.

2. Acquire data from different sources: computer simulations, physical phantoms, as well as ex vivo and in vivo tissues in order to have a large set of data for validating the proposed attenuation coefficient estimator.

3. Obtain attenuation coefficient maps with better precision compared with current attenuation coefficient estimation techniques.

\subsection{Overview of the document}

Of the most widely studied attenuation estimation methods, the spectral log difference technique has shown a more robust performance in inhomogeneous media than the spectral difference technique, and techniques based on spectral shifts such as the hybrid method have not shown to significantly outperform it. The spectral log difference technique has been used recently in many clinical studies $[11,14,18,20,21]$. Therefore, this technique was chosen to introduce the new two-dimensional ACS 
estimation approach. The rest of this document is as follows:

Chapter 2, Attenuation estimation methods describes in detail the spectral log difference (SLD) technique, as well as the proposed regularized spectral log difference technique (RSLD) [36] for attenuation coefficient maps reconstruction. In addition, the procedure for implementing the algorithm to solve the optimization problem in the RSLD technique is depicted.

Chapter 3, Simulations and experiments describes the data sets evaluated in this work. In addition, this chapter provides information of the processing parameters and the metrics used for evaluation of image quality enhancement.

Chapter 4, Testing and Validation shows the attenuation coefficient maps reconstructed for each data set as well as a discussion of the results on a case by case basis.

Chapter 5, Conclusion summarizes the most important findings of this work. 


\section{Chapter 2}

\section{Attenuation estimation methods}

\subsection{Spectral log difference technique}

The SLD technique estimates the attenuation coefficient of a data block by splitting the data block in two sub-blocks (one proximal and one distal relative to the transducer) as depicted in Fig 2.1. The centers of these windows are located at depths $z_{p}$ and $z_{d}$, respectively $\left(z_{d}>z_{p}\right)$. The backscattered power spectrum $S_{s}\left(f, z_{p}\right)$ (i.e, the power spectrum of the ultrasound signal that returns to the transducer direction) corresponding to the proximal window in the sample can be modeled as [27]

$$
S_{s}\left(f, z_{p}\right)=\left[P(f) D_{s}\left(f, z_{p}\right) B S C_{s}\left(f, z_{p}\right) A_{s}\left(f, z_{0}\right) e^{-4 \alpha_{s}(f)\left(z_{p}-z_{0}\right)}\right]
$$

where $P(f)$ denotes the scanner transfer function, $z_{0}$ is the depth at the beginning of the data block, $A_{s}\left(f, z_{0}\right)$ is the total ultrasound attenuation from the transducer to the beginning of the data block, $D_{s}\left(f, z_{p}\right)$ denotes the diffraction effects of the transducer, and $B S C_{s}\left(f, z_{p}\right)$ and $\alpha(f)$ are the backscatter and attenuation coefficients of the sub-block, respectively. Similarly, the power spectrum of the distal window can be modeled as

$$
S_{s}\left(f, z_{d}\right)=\left[P(f) D_{s}\left(f, z_{d}\right) B S C_{s}\left(f, z_{d}\right) A_{s}\left(f, z_{0}\right) e^{-4 \alpha_{s}(f)\left(z_{d}-z_{0}\right)}\right] .
$$

Combining (2.1) and (2.2) results in

$$
\begin{gathered}
Y(f)=4\left(z_{d}-z_{p}\right) \alpha_{s}(f)+\ln \left[\frac{B S C_{s}\left(f, z_{p}\right)}{B S C_{s}\left(f, z_{d}\right)}\right], \\
Y(f) \triangleq \ln \left[\left(\frac{S_{s}\left(f, z_{p}\right)}{S_{s}\left(f, z_{d}\right)}\right) /\left(\frac{D_{s}\left(f, z_{p}\right)}{D_{s}\left(f, z_{d}\right)}\right)\right] .
\end{gathered}
$$

The $\log$ ratio $Y(f)$ can be computed since $S_{s}\left(f, z_{p}\right)$ and $S_{s}\left(f, z_{d}\right)$ were measured, whereas the ratio $\left[D_{s}\left(f, z_{p}\right) / D_{s}\left(f, z_{d}\right)\right]$ can be estimated either analytically or by using reference phantom method (see section 2.2). In addition, the attenuation coefficient in soft tissues is approximated to have a linear dependence with frequency, i.e. $\alpha_{s}(f)=\beta f$, where $\beta$ is the ACS of the data block. Therefore (2.3) can be written as

$$
Y(f)=4 L \beta f+c,
$$

where $L=z_{d}-z_{p}$, and $c=\ln \left[B S C_{s}\left(f, z_{p}\right)\right]-\ln \left[B S C_{s}\left(f, z_{d}\right)\right]$. The ACS is estimated from the linear fit over the useful frequency range in (2.5). The useful frequency was selected as the frequency range that 


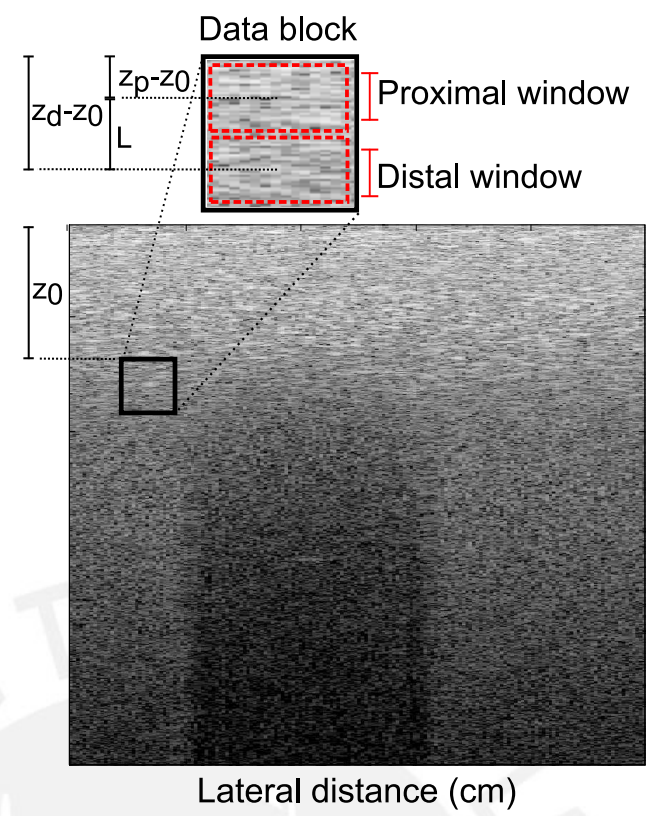

Figure 2.1: Data block beginning at depth $z_{0}$, in dashed red are depicted both proximal (center at $z_{p}$ depth) and distal (center at $z_{d}$ depth) windows.

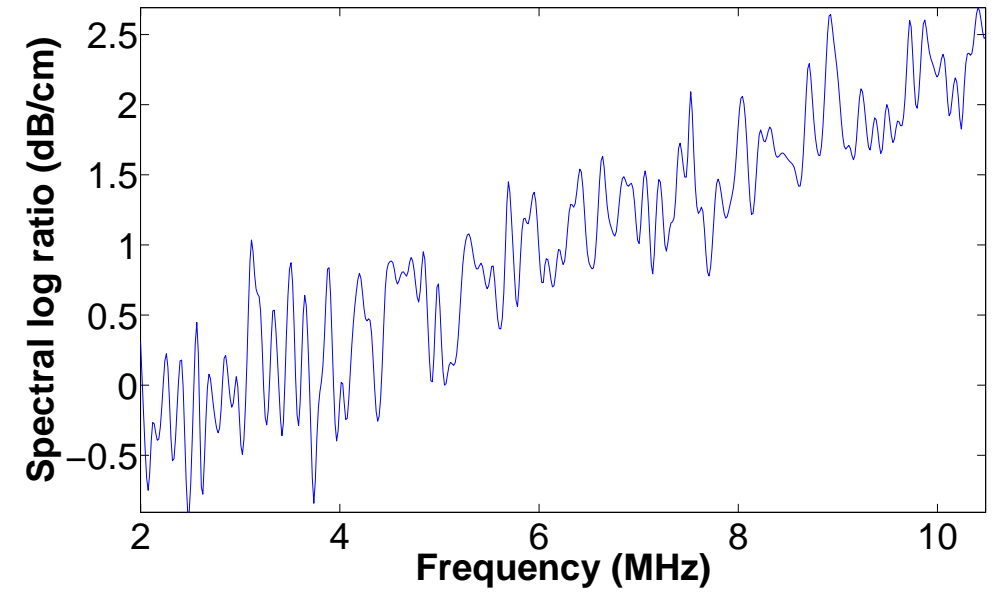

Figure 2.2: Spectral log ratio vs. frequency.

corresponds to the bandwidth of the power spectra using a -15 to $-20 \mathrm{~dB}$ cutoff criterion and that is above the noise floor (i.e., $>10 \mathrm{~dB}$ above the noise floor in this work). Figure 2.2 show an spectral $\log$ ratio for a $70 \lambda$ data block. The attenuation coefficient map is constructed by positioning the estimated ACS values in a 2D grid as shown in Fig. 2.3.

Kuc and Schwartz demonstrated that the noise in the $Y(f)$ measurements can be very closely approximated by a zero mean Gaussian PDF [5]. Therefore, a discretized form $Y_{i, j, k}$ of $Y(f)$ values in (2.5) for the data block corresponding to $(i, j)$ indices with $i \in[1, m]$ and $j \in[1, n]$, and the frequency component $f_{k}$ with $k \in[1, r]$ leads to

$$
Y_{i, j, k}=4 L \beta_{i, j} f_{k}+c_{i, j, k}+\eta_{i, j, k}
$$




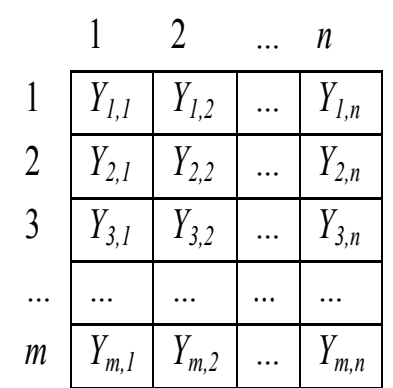

\begin{tabular}{|c|c|c|c|c|}
\hline & 1 & 2 & $\ldots$ & $n$ \\
\hline 1 & $\beta_{1,1}$ & $\beta_{1,2}$ & $\ldots$ & $\beta_{1, n}$ \\
\hline 2 & $\beta_{2,1}$ & $\beta_{2,2}$ & $\ldots$ & $\beta_{2, n}$ \\
\hline 3 & $\beta_{3,1}$ & $\beta_{3,2}$ & $\ldots$ & $\beta_{3, n}$ \\
\hline.. & $\ldots$ & $\ldots$ & $\ldots$ & $\ldots$ \\
\hline$m$ & $\beta_{m, 1}$ & $\beta_{m, 2}$ & $\ldots$ & $\beta_{m, n}$ \\
\hline
\end{tabular}

Figure 2.3: Measured log ratios and attenuation coefficient slope $\beta$ corresponding to the data blocks within the total field of view allocated in a $2 \mathrm{D}$ grid.

where $\beta_{i, j}$ is the ACS of the data block, $c_{i, j, k}$ is a term dependent on backscatter coefficients differences of proximal and distal windows, and $\eta_{i, j, k}$ is the noise that has been approximated by a zero mean Gaussian noise and were assumed to be independent and identical distributed. Following common assumptions of the SLD method, here it is considered that $c_{i, j, k}=c_{i, j}, \forall k \in[1, r]$.

\subsection{Diffraction compensation functions for the spectral log difference}

\subsubsection{Spherically focused transducers}

The ratio $\left[D_{s}\left(f, z_{p}\right) / D_{s}\left(f, z_{d}\right)\right]$ in 2.4 when using a spherically focused transducer can be estimated by computing both $D_{s}\left(f, z_{p}\right)$ and $D_{s}\left(f, z_{d}\right)$ with the diffraction compensation function developed in [37] and used previously for the SLD technique in [38], i.e.,

$$
D_{s}(f, z)= \begin{cases}0.46 \frac{\pi a^{2}}{z^{2}} \exp \left[\frac{-0.46}{\pi} G_{p}^{2}\left(\frac{F_{L}}{z}-1\right)^{2}\right],\left(\frac{1+\pi}{G_{p}}\right)^{-1}<\frac{z}{F_{L}}<\left(\frac{1-\pi}{G_{p}}\right)^{-1} \\ \frac{\pi a^{2}}{z^{2}}\left[G_{p}\left(\frac{F_{L}}{z}-1\right)\right]^{2} & \text {, otherwise }\end{cases}
$$

where $G_{p}$ is the pressure gain factor, $z$ is the depth of either the proximal or distal window, $a$ is the transducer radius, and $F_{L}$ is the transducer focal length.

\subsubsection{Linear array transducers}

The ratio $\left[D_{s}\left(f, z_{p}\right) / D_{s}\left(f, z_{d}\right)\right]$ when using linear array cannot be determined with an analytic diffraction compensation function. Thus, additional power spectra $S_{r}\left(f, z_{p}\right)$ and $S_{r}\left(f, z_{d}\right)$ of a homogeneous reference phantom with $D_{r}(f, z) \approx D_{s}(f, z)$ were acquired. The backscattered power spectra $S_{r}\left(f, z_{p}\right)$ and $S_{r}\left(f, z_{d}\right)$ are given by

$$
\begin{aligned}
& S_{r}\left(f, z_{p}\right)=\left[P(f) D_{r}\left(f, z_{p}\right) B S C_{r}\left(f, z_{p}\right) A_{r}\left(f, z_{0}\right) e^{-4 \alpha_{r}(f)\left(z_{p}-z_{0}\right)}\right], \\
& S_{r}\left(f, z_{d}\right)=\left[P(f) D_{r}\left(f, z_{d}\right) B S C_{r}\left(f, z_{d}\right) A_{r}\left(f, z_{0}\right) e^{-4 \alpha_{r}(f)\left(z_{d}-z_{0}\right)}\right] .
\end{aligned}
$$

Since the reference phantom used was homogeneous, $B S C_{r}\left(f, z_{p}\right)=B S C_{r}\left(f, z_{d}\right)$. Therefore, the ratio $\left[D_{s}\left(f, z_{p}\right) / D_{s}\left(f, z_{d}\right)\right]$ can be estimated as

$$
\frac{D_{s}\left(f, z_{p}\right)}{D_{s}\left(f, z_{d}\right)} \approx \frac{D_{r}\left(f, z_{p}\right)}{D_{r}\left(f, z_{d}\right)}=\frac{S_{r}\left(f, z_{p}\right)}{S_{r}\left(f, z_{d}\right)} e^{-4 \alpha_{r}(f)\left(z_{p}-z_{d}\right)}
$$


where the power spectra $S_{r}\left(f, z_{p}\right)$ and $S_{r}\left(f, z_{d}\right)$ are experimentally measured and the attenuation coefficient of the reference phantom $\alpha_{r}(f)$, and the proximal and distal windows depths are all known.

\subsection{Regularized spectral log difference technique}

\subsubsection{RSLD derivation}

The RSLD technique solved the ACS estimation as a two-dimensional inverse problem. By using the isotropic total variation regularization terms (assuming sparsity of spatial variations of attenuation coefficients and backscatter coefficients in the medium).

The notation used in 2.6 allows writing the SLD technique as the matrix equation

$$
y=A x+\eta,
$$

where

$$
\begin{gathered}
A \triangleq\left[\begin{array}{cccccccc}
4 L f_{1} & 0 & \cdots & 0 & 1 & 0 & \cdots & 0 \\
0 & 4 L f_{1} & \cdots & 0 & 0 & 1 & \cdots & 0 \\
\vdots & \vdots & \ddots & \vdots & \vdots & \vdots & \ddots & \vdots \\
0 & 0 & \cdots & 4 L f_{1} & 0 & 0 & \cdots & 1 \\
\vdots & \vdots & \ddots & \vdots & \vdots & \vdots & \ddots & \vdots \\
4 L f_{r} & 0 & \cdots & 0 & 1 & 0 & \cdots & 0 \\
0 & 4 L f_{r} & \cdots & 0 & 0 & 1 & \cdots & 0 \\
\vdots & \vdots & \ddots & \vdots & \vdots & \vdots & \ddots & \vdots \\
0 & 0 & \cdots & 4 L f_{r} & 0 & 0 & \cdots & 1
\end{array}\right]_{m n r \times 2 m n} \\
y \triangleq\left[\begin{array}{c}
Y_{1,1,1} \\
Y_{2,1,1} \\
\vdots \\
Y_{m, n, 1} \\
\vdots \\
Y_{1,1, r} \\
Y_{2,1, r} \\
\vdots \\
Y_{m, n, r}
\end{array}\right]_{m n r \times 1}, x \triangleq\left[\begin{array}{c}
\beta_{1,1} \\
\beta_{2,1} \\
\vdots \\
\beta_{m, n} \\
c_{1,1} \\
c_{2,1} \\
\vdots \\
c_{m, n}
\end{array}\right]_{2 m n \times 1} \quad, \eta \triangleq\left[\begin{array}{c}
\eta_{1,1,1} \\
\eta_{2,1,1} \\
\vdots \\
\eta_{m, n, 1} \\
\vdots \\
\eta_{1,1, r} \\
\eta_{2,1, r} \\
\vdots \\
\eta_{m, n, r}
\end{array}\right]_{m n r \times 1}
\end{gathered}
$$

In addition the vectors $B$ and $C$ corresponding to the upper and down halves of $x$ are defined as

$$
B \triangleq\left[\begin{array}{c}
\beta_{1,1} \\
\beta_{2,1} \\
\vdots \\
\beta_{m, n}
\end{array}\right]_{m n \times 1}, C \triangleq\left[\begin{array}{c}
c_{1,1} \\
c_{2,1} \\
\vdots \\
c_{m, n}
\end{array}\right]_{m n \times 1} .
$$

The attenuation coefficient map is obtained by reshaping the vector $B$ that groups the estimated ACS values in a $m \times n$ grid. The expression in 2.11 corresponds to the SLD method written as a matrix equation. Nevertheless, this formalism allows incorporating sparsity-driven constraints that allows 
regularizing the image reconstruction process [39]. In this work, the RSLD technique assumes physical structures within the medium to be piecewise homogeneous. Under this assumption, the regularization term chosen was the isotropic total variation [40, 41]. Hence, ACS maps can be estimated by solving

$$
\begin{gathered}
\underset{x}{\operatorname{minimize}} \frac{1}{2}\|y-A x\|_{2}^{2}+\mu \phi(x), \\
\phi(x) \triangleq \mathrm{TV}(B)+\mathrm{TV}(C),
\end{gathered}
$$

where $(1 / 2)\|y-A x\|_{2}^{2}$ is the data fidelity term, $\mu$ is the regularization parameter, and $\phi(x)$ is the regularization term. The $\operatorname{TV}(B)$ and $\operatorname{TV}(C)$ terms used in this work were

$$
\begin{aligned}
& \operatorname{TV}(B)=\sum_{i, j} \sqrt{\left(\beta_{i+1, j}-\beta_{i, j}\right)^{2}+\left(\beta_{i, j+1}-\beta_{i, j}\right)^{2}} \\
& \operatorname{TV}(C)=\sum_{i, j} \sqrt{\left(c_{i+1, j}-c_{i, j}\right)^{2}+\left(c_{i, j+1}-c_{i, j}\right)^{2}}
\end{aligned}
$$

Moreover, it should be noticed that the problem in 2.16 can be rewritten as

$$
\underset{B, C}{\operatorname{minimize}} \frac{1}{2}\left\|y-A_{1} B-A_{2} C\right\|_{2}^{2}+\mu(\operatorname{TV}(B)+\operatorname{TV}(C))
$$

where $A_{1}$ and $A_{2}$ are left and right sub-matrixes of $A$ that operate on $B$ and $C$, respectively. By defining $D \triangleq y-A_{1} B-A_{2} C,(2.17)$ can be expressed as the constrained optimization problem

$$
\begin{array}{ll}
\underset{B, C, D}{\operatorname{minimize}} & \frac{1}{2}\|D\|_{2}^{2}+\mu(\mathrm{TV}(B)+\mathrm{TV}(C)) \\
\text { subject to } & A_{1} B+A_{2} C+D=y
\end{array}
$$

\subsubsection{RSLD implementation}

The solution that minimizes (2.18) with two TV terms was achieved by using the scaled alternating direction method of multipliers (ADMM) [42]. The following ADMM algorithm was implemented

1.Set $q=0, \rho=1, B^{(0)}, C^{(0)}, D^{(0)}, V^{(0)}$

\section{Repeat}

$$
\begin{aligned}
& 3 . B^{(q+1)}=\min _{B}\left\{\mu \operatorname{TV}(B)+\frac{\rho}{2}\left\|A_{1} B+A_{2} C^{(q)}+D^{(q)}-y+V^{(q)}\right\|_{2}^{2}\right\} \\
& 4 . C^{(q+1)}=\min _{C}\left\{\mu \operatorname{TV}(C)+\frac{\rho}{2}\left\|A_{1} B^{(q+1)}+A_{2} C+D^{(q)}-y+V^{(q)}\right\|_{2}^{2}\right\} \\
& 5 . D^{(q+1)}=\min _{D}\left\{\frac{1}{2}\|D\|_{2}^{2}+\frac{\rho}{2}\left\|A_{1} B^{(q+1)}+A_{2} C^{(q+1)}+D-y+V^{(q)}\right\|_{2}^{2}\right\} \\
& 6 . V^{(q+1)}=V^{(q)}+A_{1} B^{(q+1)}+A_{2} C^{(q+1)}+D^{(q+1)}-y
\end{aligned}
$$

7.Until stopping criterion is reached.

The ADMM algorithm stops either when $\Delta\left(1 / 2\|D\|_{2}^{2}+\mu(\operatorname{TV}(B)+\operatorname{TV}(C))\right.$ between consecutive iterations is smaller than a tolerance value (i.e., $10^{-3}$ in this work) or the maximum number of iterations is reached (i.e., 20 iterations in this work). The sub-problems 3 and 4 in the ADMM algorithm that involve the minimization of a cost function with a single TV term were independently solved by using the iterative reweighted least squares (IRLS) algorithm described in [40]. 


\section{Chapter 3}

\section{Simulations and experiments}

The quality of ACS maps when using the RSLD technique was numerically evaluated on numerical phantoms, physical phantoms, and ex vivo and in vivo tissues. The data processing was performed off-line using the software MATLAB v.8.3 (The MathWorks Inc., Natick, MA)

\subsection{Data sets}

\subsubsection{Computer simulations}

The computer simulations were performed using the k-Wave toolbox [43], which provides time-domain simulations accounting for both linear and nonlinear wave propagation and arbitrary distributions of material parameters. For all simulations, the simulated array transducer was composed by 128 elements with a $0.3 \mathrm{~mm}$ element pitch. The focal number in transmit and receive was set to two. Fixed focusing was used in transmission (focal depth of $2 \mathrm{~cm}$, focal number of 2), and dynamic focusing was used on reception. The excitation signal was a Gaussian pulse with $6.66 \mathrm{MHz}$ central frequency and $50 \%$ full width at half maximum (FWHM). For both computer simulations, the diffractive effects of the transducers were compensated with a simulated homogeneous reference phantom with ACS of 0.30 $\mathrm{dB} \cdot \mathrm{cm}^{-1} \cdot \mathrm{MHz}^{-1}$.

The numerical phantom in Simulation \#1 consisted of a homogeneous medium with ACS of $0.50 \mathrm{~dB} . \mathrm{cm}-1 . \mathrm{MHz}-1$, which is typically considered as a representative value for soft tissues [44]. The numerical phantom in Simulation \#2 was a homogeneous background with a $2 \mathrm{~cm}$ diameter circular inclusion. The ACS values in the background and inclusion were 0.5 and $1 \mathrm{~dB} . \mathrm{cm}^{-1} \cdot \mathrm{MHz}^{-1}$. Figure 3.1 shows the inhomogeneous attenuation coefficient map used for simulation radiofrequency backscattered echoes.

\subsubsection{Tissue-mimicking phantom}

Two agar-based tissue-mimicking phantoms were used in this study. Phantom \#1 was an agar-based phantom designed with a cylindrical shape consisting of a $70 \mathrm{~mm}$ diameter background with a 25 $\mathrm{mm}$ inclusion. Attenuation properties of background and inclusion regions were set by using different concentrations of graphite powder [45]. The ground truth ACS values were found to be 0.54 and 1.04 $\mathrm{dB} . \mathrm{cm}^{-1} \cdot \mathrm{MHz}^{-1}$ in the background and inclusion regions, respectively, using insertion loss techniques. Phantom \#2 was constructed with the same dimensions as the Phantom \#1 with ACS that were found to be 0.41 and $0.75 \mathrm{~dB} \cdot \mathrm{cm}^{-1} \cdot \mathrm{MHz}^{-1}$ in the background and inclusion regions, respectively, using insertion 


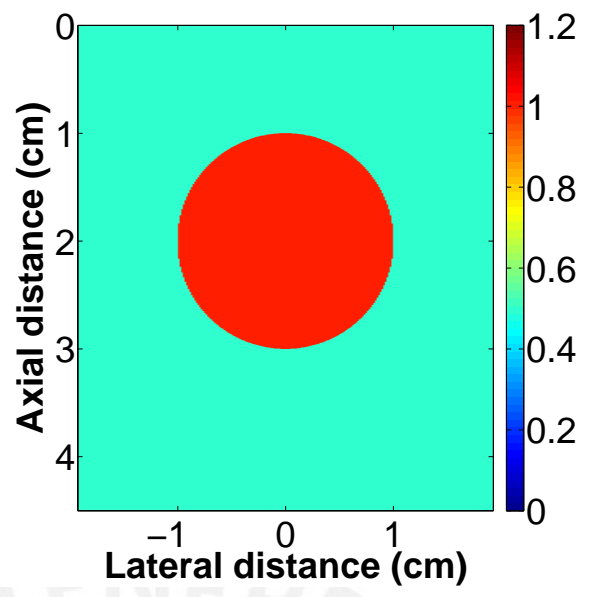

Figure 3.1: Inhomogeneous attenuation coefficient map in $\mathrm{dB} \cdot \mathrm{cm}^{-1} \cdot \mathrm{MHz}^{-1}$ used for simulating radiofrequency backscattered echoes.

loss techniques.

Data acquisition was performed using a micro-positioning system controlled by custom LabVIEW software (National Instruments, Austin, TX). A 7.5 MHz (f/4) single element transducer driven by a Olympus Panametrics 5900 pulser/receiver (Olympus Corporation, Waltham, MA) as well as a UF34121 14-bit digitizer PCI-X card with a $250 \mathrm{MHz}$ sampling frequency (Strategic Test Corporation, Woburn, MA) was used to collect the data. The transducer was moved along the lateral axis with a $0.5 \mathrm{~mm}$ step. The diffractive effects of the single element transducer were computed analytically using (2.7).
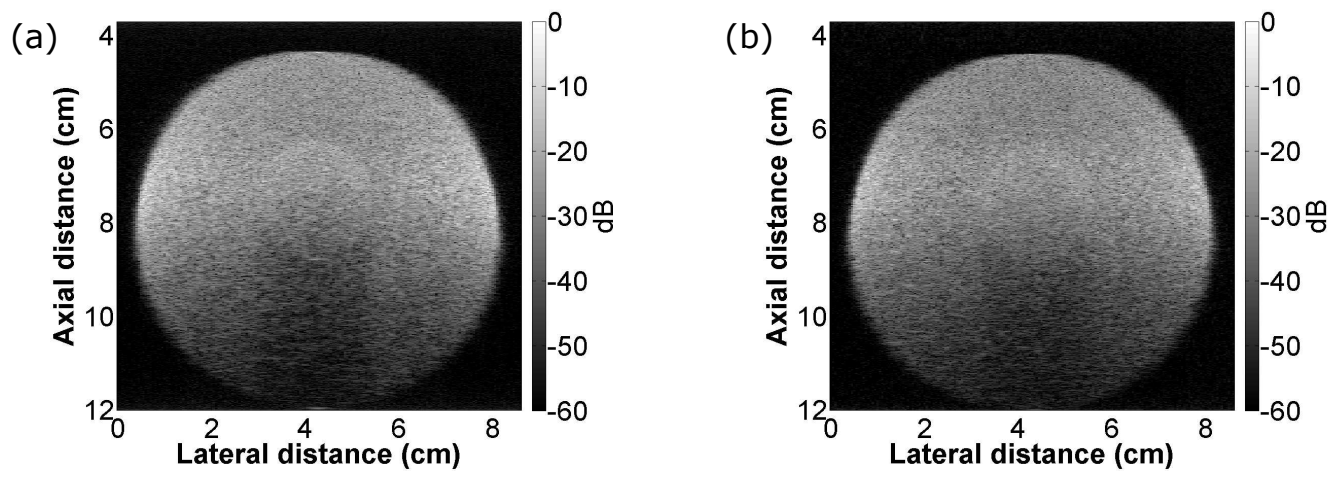

Figure 3.2: B-mode images of Phantom \#1 (a) and Phantom \#2 (b).

\subsubsection{Ex vivo tissue}

A piece of ex vivo chicken breast tissue was embedded into an agar, $70 \mathrm{~mm}$ diameter cylindrical background. The procedure for data acquisition for ex vivo tissue was the same used for the physical phantom in Section 3.1.2. Figure 3.3 shows the procedure for the data acquistion from the ex vivo chicken breast tissue embedded into an agar background. The ground truth ACS value of the chicken breast sample estimated using insertion loss techniques was found to be $1.24 \mathrm{~dB} \cdot \mathrm{cm}^{-1} \cdot \mathrm{MHz}^{-1}$. 


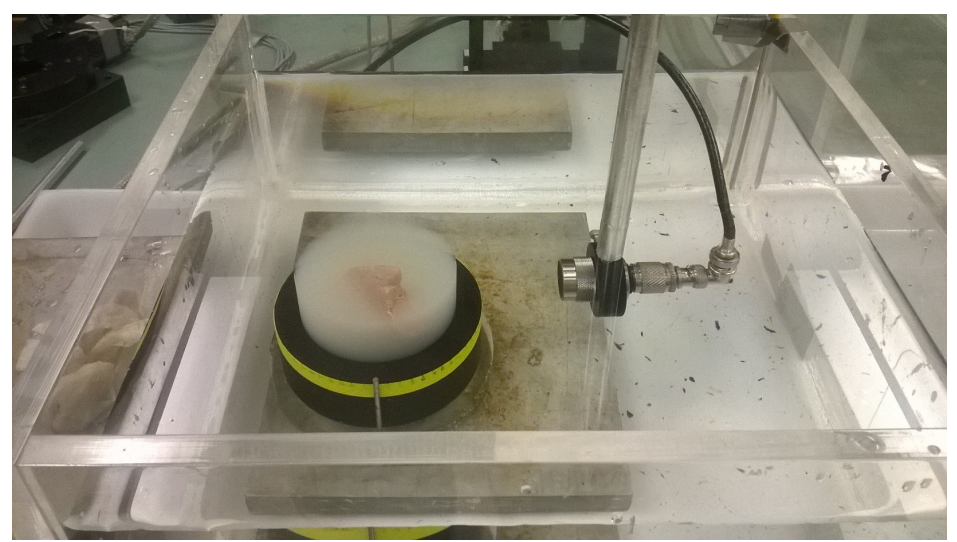

Figure 3.3: Procedure for the data acquistion from the ex vivo chicken breast tissue embedded into an agar background.

\subsubsection{In vivo tissue}

The in vivo data of a breast tumor was acquired in an oncology clinic (Oncosalud, Lima, Peru) following procedures of informed consent. The data acquisition was performed using a SonixTouch ultrasound scanner (Analogic Ultrasound, Peabody, MA) with an L14-5 linear array transducer (nominal center frequency of $10 \mathrm{MHz}$ ). The diffractive effects of the transducer were compensated using a reference phantom [46] containing embedded $18.2 \pm 1 \mu \mathrm{m}$ diameter spherical glass beads randomly distributed in an mboxagar-based matrix at a concentration of 78.12 spheres per $\mathrm{mm}^{3}$. A polynomial regression was employed to fit the experimentally estimated attenuation coefficient of the reference phantom between 1 and $20 \mathrm{MHz}, \alpha_{r e f}(f)=0.0057 f^{2}+0.4432 f-0.1$, where $f$ is the ultrasonic frequency in $\mathrm{MHz}$ and $\alpha_{\text {ref }}$ is the attenuation coefficient of the reference phantom in $\mathrm{dB} \cdot \mathrm{cm}^{-1}$. In a mammographic examination conducted prior to the ultrasound data acquisition, the mammary density of the breast was found to be heterogeneously dense (i.e., both fibroglandular and fat tissues are present but fibroglandular tissue is more prevalent). The results from a biopsy performed after ultrasound data acquisition determined the tumor to be a fibroadenoma. Figure 3.4 shows the B-mode image of the itin vivo breast sample used.

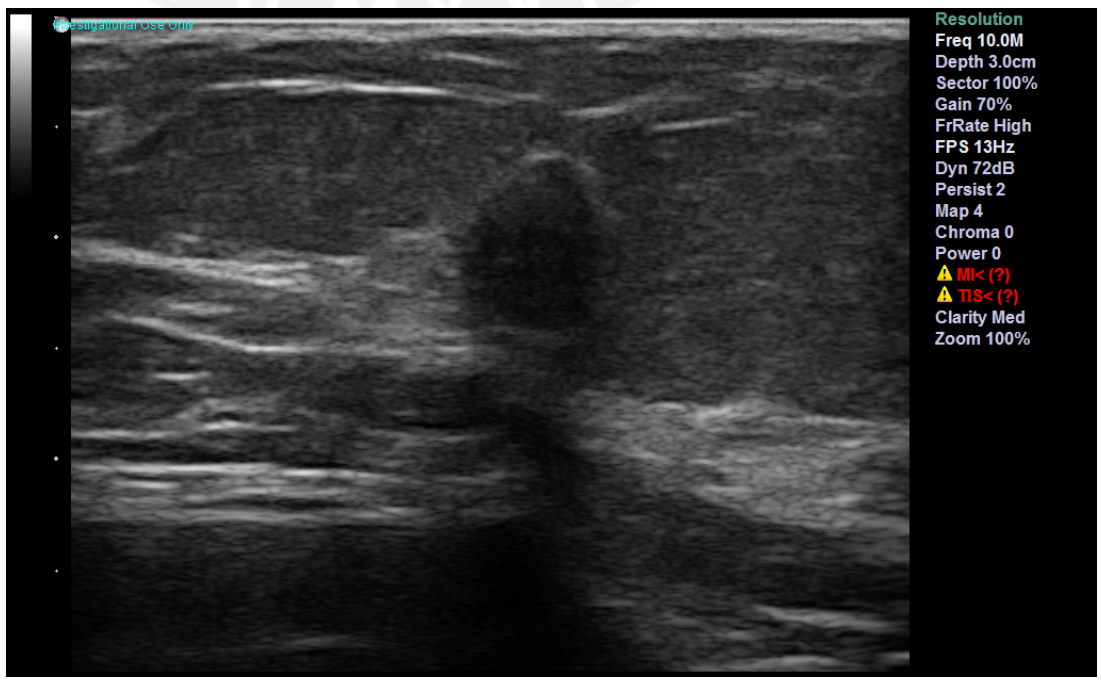

Figure 3.4: B-mode image of in vivo breast tumor. 


\subsection{Construction of attenuation coefficient maps}

The field of view was selected so that it contained the inclusion region and part of the background region. Several square data blocks with $80 \%$ overlap in both lateral and axial directions were outlined in the field of view. Up to five data block sizes were evaluated $(50 \lambda \times 50 \lambda, 40 \lambda \times 40 \lambda, 30 \lambda \times 30 \lambda$, $20 \lambda \times 20 \lambda$ and $10 \lambda \times 10 \lambda$ ) for reconstructing attenuation coefficient maps with both the SLD and the RSLD techniques. Table 3.1 presents the useful frequency range and wavelength sizes used for each data set.

Table 3.1: Frequency range and wavelength size of the data sets used in this work for testing the SLD and RSLD techniques.

\begin{tabular}{|c|c|c|}
\hline \multirow{2}{*}{ Data set } & \multicolumn{2}{|c|}{ ACS estimation parameters } \\
\cline { 2 - 3 } & Freq. range (MHz) & Wavelength $(\mathrm{mm})$ \\
\hline \hline Simulation \#1 & $3.3-8.7$ & 0.26 \\
\hline Simulation \#2 & $3.3-8.7$ & 0.26 \\
\hline Phantom \#1 & $2-9$ & 0.27 \\
\hline Phantom \#2 & $3-10$ & 0.23 \\
\hline Ex vivo tissue & $2-9$ & 0.27 \\
\hline In vivo tissue & $4-11$ & 0.21 \\
\hline
\end{tabular}

The regularization parameter used for constructing all attenuation coefficient maps presented in this work using the RSLD technique was $\mu=10^{2.5}$. This value was found to provide a good balance between accuracy (i.e., comparable levels of bias) and precision (i.e., the spatial variance is reduced significantly) of the ACS values. For both SLD and RSLD techniques, the attenuation coefficient images were generated using bilinear interpolation of the ACS values estimated for each data block and overlaid on top of the corresponding B-mode images.

\subsection{Evaluation of attenuation coefficient maps}

\subsubsection{Mean and standard deviation}

The mean and standard deviation (SD) of the estimated ACS values were estimated for regions manually selected within the inclusion $\left(\Omega_{i n}\right)$ and background $\left(\Omega_{b g}\right)$ regions of the attenuation coefficient maps.

\subsubsection{Percentage error}

The mean and standard deviation of the percentage error was used to evaluate the accuracy and precision of estimated ACS values in the attenuation coefficient maps, respectively. The mean percentage error (MPE) and the standard deviation of the percentage error (SDPE) were computed as

$$
\begin{gathered}
\text { MPE }=\frac{1}{Q} \times \sum_{(i, j) \in \Omega}^{Q} \frac{\beta_{i, j}-\beta_{i, j}^{*}}{\beta_{i, j}^{*}}, \\
\mathrm{SDPE}=\sqrt{\frac{1}{Q} \times \sum_{(i, j) \in \Omega}^{Q}\left(\frac{\beta_{i, j}-\beta_{i, j}^{*}}{\beta_{i, j}^{*}}-\mathrm{MPE}\right)^{2}},
\end{gathered}
$$


where $\beta_{i, j}$ and $\beta_{i, j}^{*}$ are the estimated and ground truth ACSs, respectively, in the ACS image pixel with index $(i, j)$ inside the region $\Omega$, and $Q$ is the total number of pixels in the ACS image used in the computation of the MPE and SDPE.

\subsubsection{Contrast-to-noise ratio}

The contrast-to-noise ratio (CNR) for the attenuation coefficient maps in was computed similar to the expression used in shear wave speed imaging [47], given by

$$
\mathrm{CNR}=\frac{\left|\bar{X}_{i n}-\bar{X}_{b g}\right|}{\sqrt{\sigma_{i n}^{2}+\sigma_{b g}^{2}}}
$$

where $\bar{X}$ ans $\sigma$ are the mean and standard deviation of the ACS values within the inclusion $\left(\Omega_{\text {in }}\right)$ and background $\left(\Omega_{b g}\right)$. 


\section{Chapter 4}

\section{Testing and Validation}

\subsection{Computer simulations}

\subsubsection{Homogeneous simulation}

The ACS maps of Simulation \# 1 with a homogeneous phantom obtained when using the SLD and the $\operatorname{RSLD}\left(\mu=10^{2.5}\right)$ techniques for data block sizes of $50 \lambda \times 50 \lambda, 30 \lambda \times 30 \lambda$ and $20 \lambda \times 20 \lambda$ are shown in Fig. 4.1. Table 4.1 presents the mean, standard deviation, MPE and SDPE calculated within a $115 \lambda \times 145 \lambda$ region.

As it can be observed in Fig. 4.1, the ACS maps reconstructed using the SLD suffered a dramatic increase in variance as the data block size was reduced. When using SLD, standard deviations of the percentage error less than $20 \%$ were only obtained for data blocks of size $50 \lambda \times 50 \lambda$. Considering that the pulse duration was $0.67 \mu \mathrm{s}$, the pulse length was $1.03 \mathrm{~mm}$ and therefore the axial extent of the data block was approximately 11.25 pulse lengths. Also, the number of effective uncorrelated lines $N_{\text {eff }}$ within a data block can be calculated with [48]

$$
N_{\text {eff }}=N\left[1+\frac{2}{N} \sum_{\Delta=1}^{N-1}(N-\Delta)(\gamma(\Delta))\right]^{-1}
$$

where $N$ is the total number of lines inside the block, and $\gamma(\Delta)$ is the correlation coefficient between rf data segments that are separated by $\Delta$ lines. Using $4.1, N_{\text {eff }}$ was found to be 32 lines for the $50 \lambda \times 50 \lambda$ data block size. Therefore, the SDPE found in this simulation is consistent with the results in [27], where a SDPE $<20 \%$ could be achieved when using a data block size of 40 uncorrelated lines laterally by 10.5 pulse lengths axially. In contrast, when using the RSLD technique the SDPE was less than $20 \%$ for data block sizes of down to $30 \lambda \times 30 \lambda$ ( 6.75 pulse lengths axially by 16 uncorrelated lines laterally), and only increased to $22 \%$ when considering a data block size of $20 \lambda \times 20 \lambda$ ( 4.5 pulse lengths axially by 9 uncorrelated lines laterally). Therefore, the proposed method provided a dramatic performance improvement when estimating ACSs in homogeneous media.

\subsubsection{Inhomogeneous simulation}

The ACS maps of Simulation \#2 with an inhomogeneous phantom obtained when using the SLD and the $\operatorname{RSLD}\left(\mu=10^{2.5}\right)$ techniques for data block sizes $40 \lambda \times 40 \lambda, 30 \lambda \times 30 \lambda$ and $20 \lambda \times 20 \lambda$ are shown in Fig. 4.2. Table 4.2 presents the mean, standard deviation, MPE and SDPE calculated within a $50 \lambda \times 50 \lambda$ region inside the inclusion. 


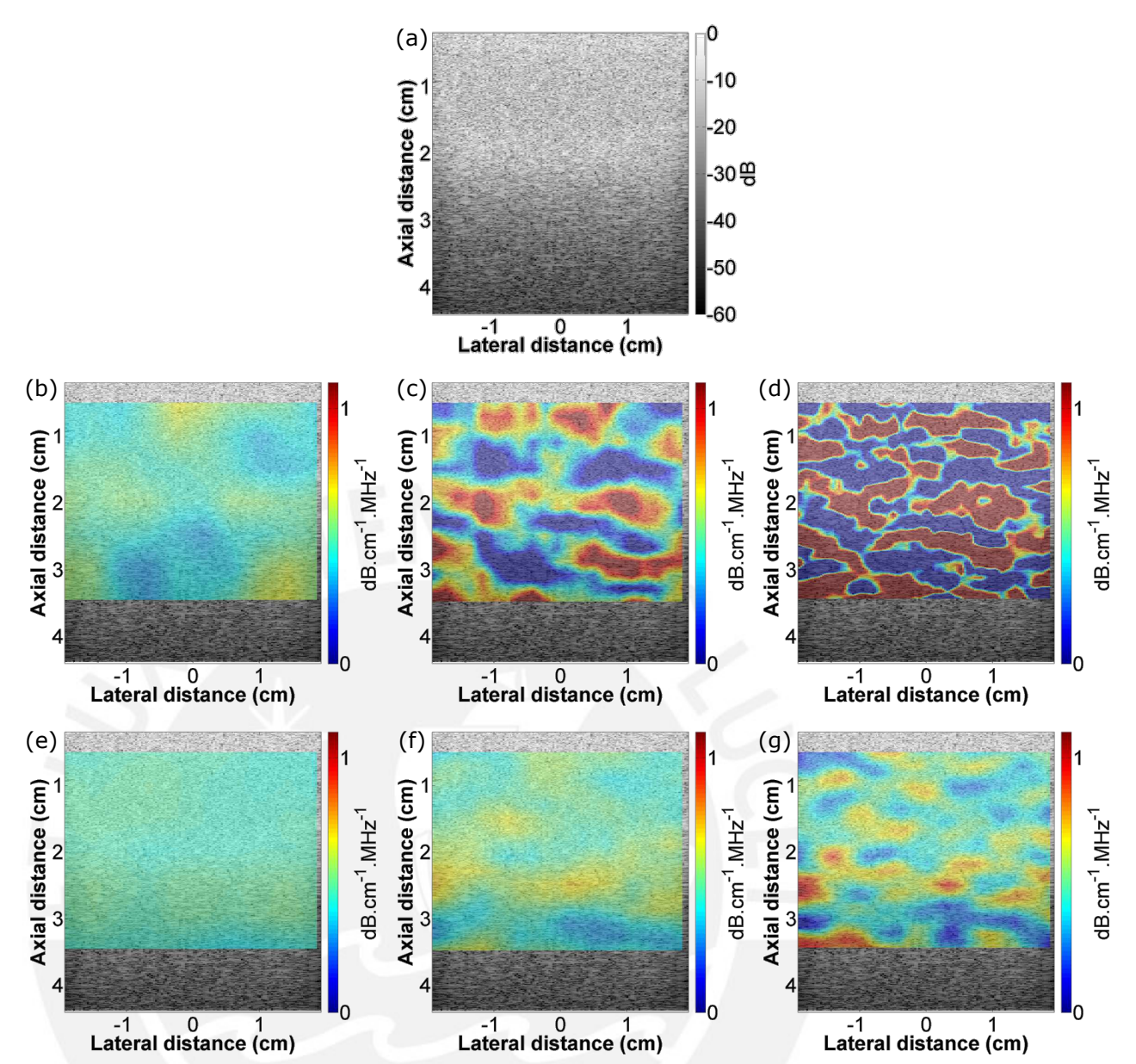

Figure 4.1: B-mode image for Simulation \#1 (a) and ACS maps when using the SLD (b)-(d) and RSLD (e)-(g) techniques for data block sizes of $50 \lambda \times 50 \lambda, 30 \lambda \times 30 \lambda$ and $20 \lambda \times 20 \lambda$, respectively.

Table 4.1: Estimated ACS mean, standard deviation, MPE and SDPE for Simulation \#1 obtained when using the SLD and the RSLD techniques. The ground truth ACS value was $0.50 \mathrm{db} \cdot \mathrm{cm}^{-1} \cdot \mathrm{MHz}^{-1}$.

\begin{tabular}{|c|c|c|c|c|c|c|}
\hline $\begin{array}{c}\text { Data block } \\
\text { size }(\lambda)\end{array}$ & \multicolumn{2}{|c|}{ Mean \pm SD $\left(\mathrm{db}^{-1} \cdot \mathrm{MHz}^{-1}\right)$} & \multicolumn{2}{|c|}{ MPE } & \multicolumn{2}{c|}{ SDPE } \\
\cline { 2 - 7 } & SLD & RSLD & SLD & RSLD & SLD & RSLD \\
\hline \hline $50 \times 50$ & $0.47 \pm 0.07$ & $0.48 \pm 0.02$ & $6 \%$ & $3 \%$ & $14 \%$ & $5 \%$ \\
\hline $40 \times 40$ & $0.48 \pm 0.16$ & $0.49 \pm 0.05$ & $5 \%$ & $2 \%$ & $31 \%$ & $9 \%$ \\
\hline $30 \times 30$ & $0.50 \pm 0.34$ & $0.52 \pm 0.08$ & $0 \%$ & $3 \%$ & $67 \%$ & $16 \%$ \\
\hline $20 \times 20$ & $0.51 \pm 0.98$ & $0.51 \pm 0.15$ & $2 \%$ & $2 \%$ & $197 \%$ & $30 \%$ \\
\hline $10 \times 10$ & $0.48 \pm 2.15$ & $0.48 \pm 0.16$ & $3 \%$ & $4 \%$ & $429 \%$ & $33 \%$ \\
\hline
\end{tabular}

The drawbacks of ACS imaging with the SLD technique can be more clearly observed in the inhomogeneous Simulation \#2. Using small data block sizes such as $20 \lambda \times 20 \lambda$ or $10 \lambda \times 10 \lambda$ resulted in large spatial variance of the ACS maps (i.e., SDPE $>70 \%$ ), which complicated the detection of the inclusion. The results show that the RSLD technique yielded comparable accuracy levels than the SLD technique when using large data blocks. However, the RSLD technique generated ACS maps with 

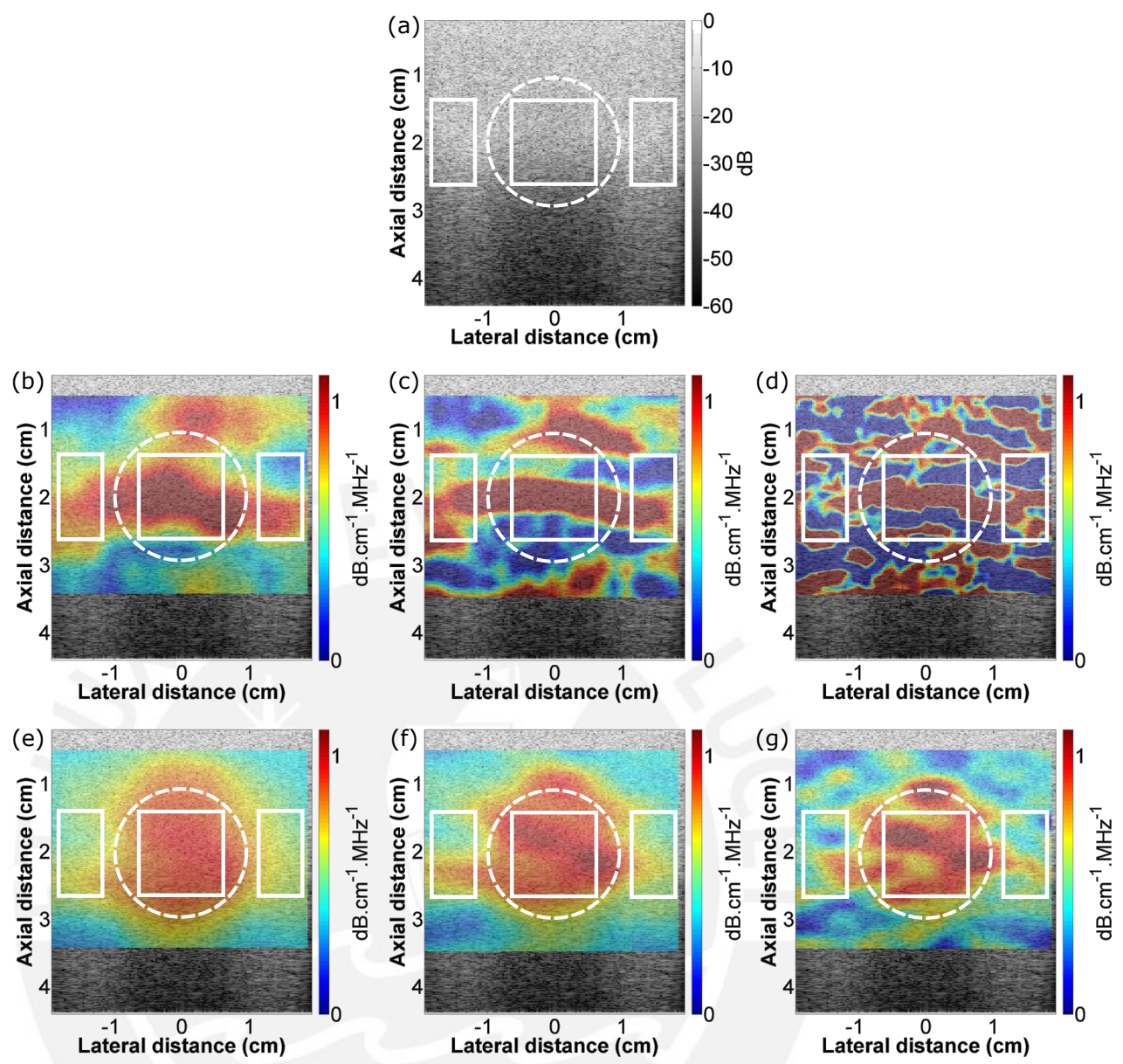

Figure 4.2: B-mode image for Simulation \#2 (a) and ACS maps when using the SLD (b)-(d) and RSLD (e)-(g) techniques, respectively, for data block sizes of $40 \lambda \times 40 \lambda, 30 \lambda \times 30 \lambda$ and $20 \lambda \times 20 \lambda$. The dashed and solid lines outline the inclusion and regions for the calculation of performance metrics, respectively.

both MPE and SDPE below $15 \%$ for data block sizes of $30 \lambda \times 30 \lambda$ or smaller. Therefore, the RSLD technique allowed better identification of inclusions in simulations.

Table 4.2: Estimated ACS mean, standard deviation, MPE and SDPE for Simulation \#2 obtained when using the SLD and the RSLD techniques. The ground truth ACS value was $1.0 \mathrm{db} \cdot \mathrm{cm}^{-1} \cdot \mathrm{MHz}^{-1}$.

\begin{tabular}{|c|c|c|c|c|c|c|}
\hline \multirow{2}{*}{$\begin{array}{c}\text { Data block } \\
\text { size }(\lambda)\end{array}$} & \multicolumn{2}{|c|}{ Mean $\pm \mathrm{SD}\left(\mathrm{db}^{\left.-\mathrm{cm}^{-1} . \mathrm{MHz}^{-1}\right)}\right.$} & \multicolumn{2}{c|}{ MPE } & \multicolumn{2}{c|}{ SDPE } \\
\cline { 2 - 7 } & SLD & RSLD & SLD & RSLD & SLD & RSLD \\
\hline \hline $50 \times 50$ & $0.86 \pm 0.11$ & $0.84 \pm 0.04$ & $14 \%$ & $16 \%$ & $11 \%$ & $4 \%$ \\
\hline $40 \times 40$ & $0.96 \pm 0.23$ & $0.92 \pm 0.04$ & $4 \%$ & $8 \%$ & $23 \%$ & $4 \%$ \\
\hline $30 \times 30$ & $0.81 \pm 0.60$ & $0.94 \pm 0.06$ & $19 \%$ & $6 \%$ & $60 \%$ & $6 \%$ \\
\hline $20 \times 20$ & $0.75 \pm 1.24$ & $0.92 \pm 0.13$ & $25 \%$ & $8 \%$ & $124 \%$ & $13 \%$ \\
\hline $10 \times 10$ & $0.69 \pm 2.20$ & $0.96 \pm 0.14$ & $31 \%$ & $4 \%$ & $220 \%$ & $14 \%$ \\
\hline
\end{tabular}




\subsection{Tissue-mimicking phantom}

\subsubsection{Physical phantom \#1}

The ACS maps of the Phantom \#1 obtained with the SLD and the RSLD $\left(\mu=10^{2.5}\right)$ techniques for data block sizes $40 \lambda \times 40 \lambda, 30 \lambda \times 30 \lambda$ and $20 \lambda \times 20 \lambda$ are shown in Fig. 4.3. Table 4.3 presents the mean and standard deviation, MPE and SDPE calculated within a $60 \lambda \times 60 \lambda$ region inside the inclusion.

Table 4.3: Estimated ACS mean, standard deviation, MPE and SDPE of inclusion for Phantom \#1 obtained when using the SLD and the RSLD techniques. The ground truth ACS value was 1.04 db.cm ${ }^{-1} \cdot \mathrm{MHz}^{-1}$.

\begin{tabular}{|c|c|c|c|c|c|c|}
\hline $\begin{array}{c}\text { Data block } \\
\text { size }(\lambda)\end{array}$ & \multicolumn{2}{|c|}{ Mean $\pm \mathrm{SD}\left(\mathrm{db}^{\left.-\mathrm{cm}^{-1} . \mathrm{MHz}^{-1}\right)}\right.$} & \multicolumn{2}{c|}{ MPE } & \multicolumn{2}{c|}{ SDPE } \\
\cline { 2 - 7 } & SLD & RSLD & SLD & RSLD & SLD & RSLD \\
\hline \hline $50 \times 50$ & $0.85 \pm 0.19$ & $0.87 \pm 0.03$ & $18 \%$ & $16 \%$ & $18 \%$ & $3 \%$ \\
\hline $40 \times 40$ & $0.87 \pm 0.27$ & $0.87 \pm 0.04$ & $16 \%$ & $16 \%$ & $26 \%$ & $4 \%$ \\
\hline $30 \times 30$ & $0.90 \pm 0.37$ & $0.86 \pm 0.07$ & $14 \%$ & $17 \%$ & $35 \%$ & $7 \%$ \\
\hline $20 \times 20$ & $0.87 \pm 0.07$ & $0.87 \pm 0.10$ & $17 \%$ & $17 \%$ & $67 \%$ & $9 \%$ \\
\hline $10 \times 10$ & $0.69 \pm 2.20$ & $0.74 \pm 0.11$ & $34 \%$ & $29 \%$ & $212 \%$ & $10 \%$ \\
\hline
\end{tabular}

\subsubsection{Physical phantom \#2}

The ACS maps of the Phantom \#2 obtained with the SLD and the RSLD $\left(\mu=10^{2.5}\right)$ techniques for data block sizes $40 \lambda \times 40 \lambda, 30 \lambda \times 30 \lambda$ and $20 \lambda \times 20 \lambda$ are shown in Fig. 4.4. Table 4.4 presents the mean and standard deviation, MPE and SDPE calculated within a $60 \lambda \times 60 \lambda$ region inside the inclusion.

Table 4.4: Estimated ACS mean, standard deviation, MPE and SDPE of inclusion for Phantom \#2 obtained when using the SLD and the RSLD techniques. The ground truth ACS value was 0.75 db. $\mathrm{cm}^{-1} \cdot \mathrm{MHz}^{-1}$.

\begin{tabular}{|c|c|c|c|c|c|c|}
\hline \multirow{2}{*}{$\begin{array}{c}\text { Data block } \\
\text { size }(\lambda)\end{array}$} & \multicolumn{2}{|c|}{ Mean $\pm \mathrm{SD}\left(\mathrm{db}^{-} \mathrm{cm}^{-1} . \mathrm{MHz}^{-1}\right)$} & \multicolumn{2}{|c|}{ MPE } & \multicolumn{2}{c|}{ SDPE } \\
\cline { 2 - 7 } & SLD & RSLD & SLD & RSLD & SLD & RSLD \\
\hline \hline $50 \times 50$ & $0.7 \pm 0.1$ & $0.67 \pm 0.03$ & $6 \%$ & $11 \%$ & $13 \%$ & $4 \%$ \\
\hline $40 \times 40$ & $0.72 \pm 0.15$ & $0.67 \pm 0.04$ & $4 \%$ & $10 \%$ & $20 \%$ & $5 \%$ \\
\hline $30 \times 30$ & $0.74 \pm 0.24$ & $0.68 \pm 0.07$ & $1 \%$ & $10 \%$ & $32 \%$ & $10 \%$ \\
\hline $20 \times 20$ & $0.7 \pm 0.54$ & $0.66 \pm 0.12$ & $7 \%$ & $12 \%$ & $72 \%$ & $16 \%$ \\
\hline $10 \times 10$ & $0.71 \pm 1.81$ & $0.64 \pm 0.15$ & $5 \%$ & $14 \%$ & $241 \%$ & $21 \%$ \\
\hline
\end{tabular}

Results obtained with the physical phantoms follow similar trends to the ones observed in Simulation \#2, i.e., when using the SLD technique very large estimation variance was observed when using small data block sizes. The use of RSLD allowed reconstructing ACS maps with improved precision and accuracy. Data set from Phantom \#1 has previously been used for attenuation imaging using full angular spatial compounding (FASC) of ACS maps produced using the SLD technique with a data block size of approximately $30 \lambda \times 30 \lambda$ [33]. The use of SLD + FASC with 30 incidence angles uniformly distributed over $360^{\circ}$ resulted in a variance reduction of $88 \%$ in the inclusion region when compared to SLD alone, with an estimation bias of $8 \%$. In this work, the use of RSLD with $30 \lambda \times 30 \lambda$ data blocks resulted in a larger variance reduction (i.e., 96\%) at the expense of a slightly larger bias (i.e., 17\%). Therefore, the 

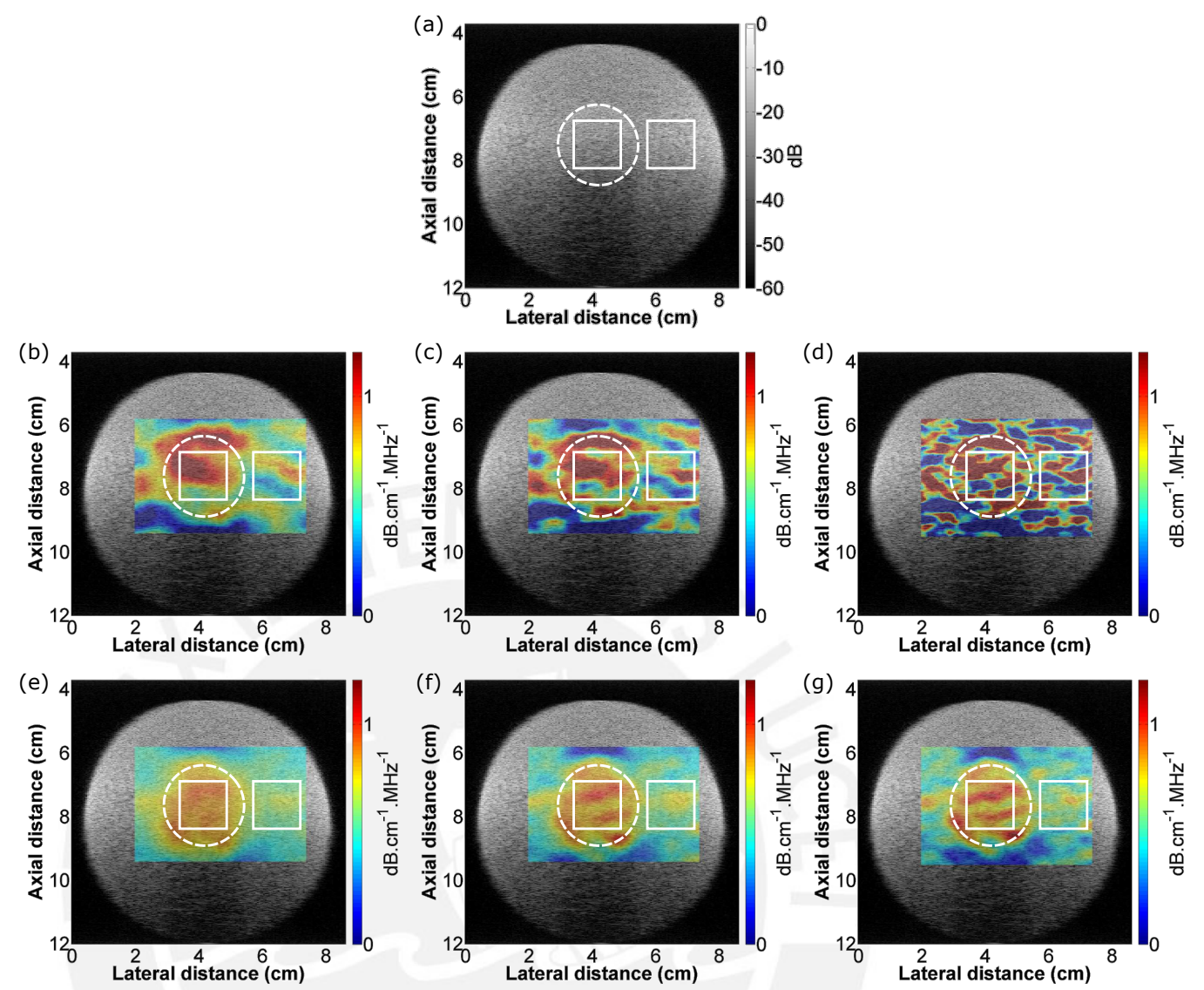

Figure 4.3: B-mode image for Phantom\#1 (a) and ACS maps when using the SLD (b)-(d) and RSLD (e)-(g) techniques, respectively, for data block sizes of $40 \lambda \times 40 \lambda, 30 \lambda \times 30 \lambda$ and $20 \lambda \times 20 \lambda$. The dashed and solid lines outline the inclusion and regions for the calculation of performance metrics, respectively.

RSLD technique has the potential to provide comparable improvements in ACS estimation precision than FASC without being limited to use with scanners capable of full angular acquisitions.

\subsection{Ex vivo tissue}

Further results were obtained using an ex vivo chicken breast sample embedded in an agar matrix. The ACS maps of the ex vivo chicken breast tissue obtained when using the SLD and the RSLD $\left(\mu=10^{2.5}\right)$ techniques for data block sizes $40 \lambda \times 40 \lambda, 30 \lambda \times 30 \lambda$ and $20 \lambda \times 20 \lambda$ are shown in Fig. 4.5. Table 4.5 presents the mean, standard deviation, MPE and SDPE calculated within a $26 \lambda \times 52 \lambda$ region inside the inclusion.

The ground truth ACS value obtained for the chicken breast tissue using insertion loss methods was $1.24 \mathrm{~dB} \cdot \mathrm{cm}^{-1} \cdot \mathrm{MHz}^{-1}$. Plots of attenuation coefficients vs. frequency in chicken breast muscle were reported by Hete and Shung [49] in the range of 5-12 MHz. The data points in the range of 5-9 MHz (i.e., the common analysis frequency band between this work and [49]) were mined using the DataThief III software and fitted to a linear function, resulting in an ACS value of $1.24 \pm 0.10$ $\mathrm{dB} . \mathrm{cm}^{-1} \cdot \mathrm{MHz}^{-1}$. Therefore, the ground truth value was in good agreement with previous reports in the 

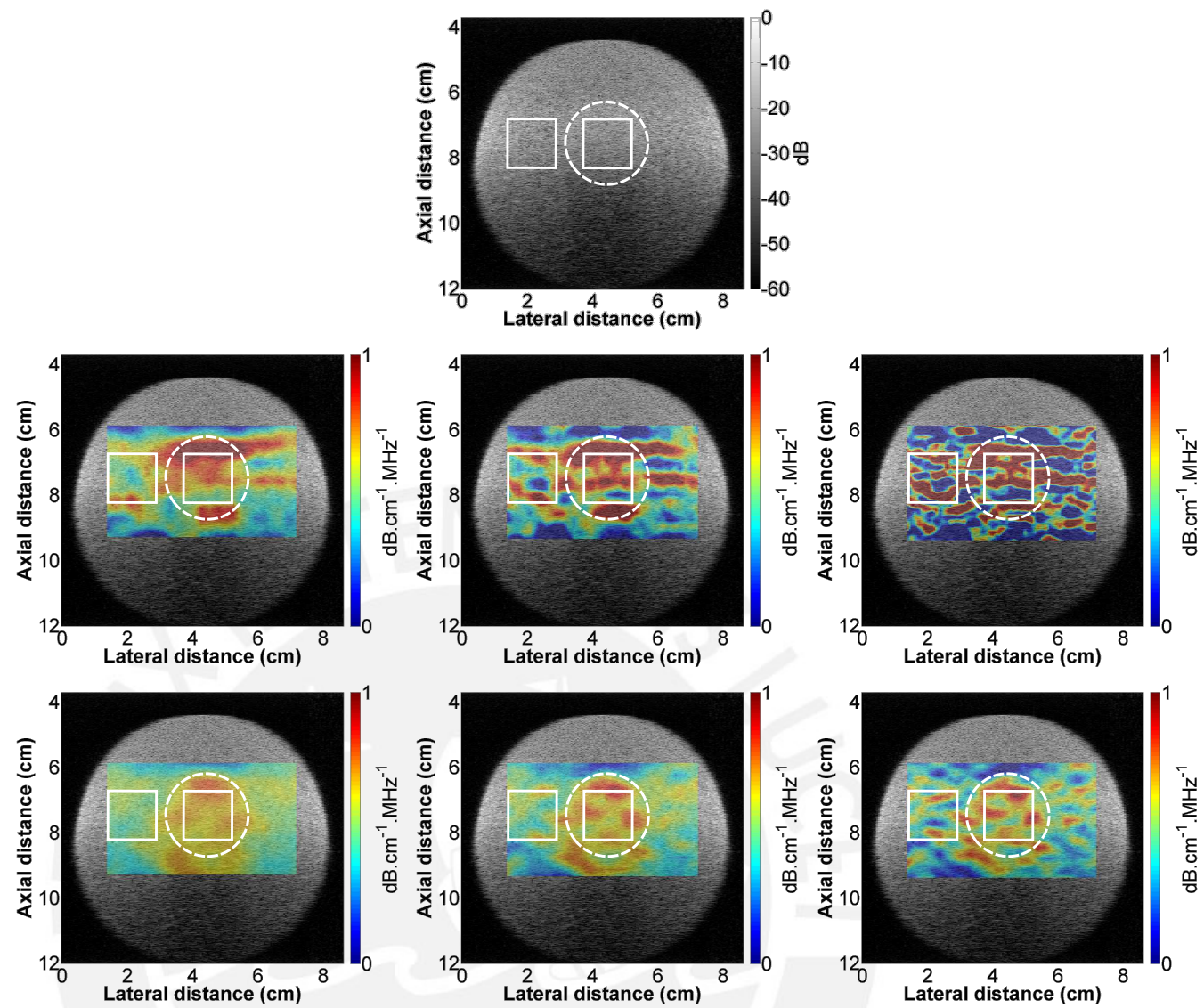

Figure 4.4: B-mode image for Phantom\#2 (a) and ACS maps when using the SLD (b)-(d) and RSLD (e)-(g) techniques, respectively, for data block sizes of $40 \lambda \times 40 \lambda, 30 \lambda \times 30 \lambda$ and $20 \lambda \times 20 \lambda$. The dashed and solid lines outline the inclusion and regions for the calculation of performance metrics, respectively.

Table 4.5: Estimated ACS mean, standard deviation, MPE and SDPE of ex vivo chicken breast tissue obtained when using the SLD and the RSLD techniques. The ground truth ACS value was 1.24 $\mathrm{db} . \mathrm{cm}^{-1} \cdot \mathrm{MHz}^{-1}$.

\begin{tabular}{|c|c|c|c|c|c|c|}
\hline $\begin{array}{c}\text { Data block } \\
\text { size }(\lambda)\end{array}$ & \multicolumn{2}{|c|}{ Mean \pm SD $\left(\mathrm{db}^{\left.-\mathrm{cm}^{-1} . \mathrm{MHz}^{-1}\right)}\right.$} & \multicolumn{2}{c|}{ MPE } & \multicolumn{2}{c|}{ SDPE } \\
\cline { 2 - 7 } & SLD & RSLD & SLD & RSLD & SLD & RSLD \\
\hline \hline $50 \times 50$ & $1.35 \pm 0.17$ & $1.23 \pm 0.03$ & $9 \%$ & $1 \%$ & $13 \%$ & $3 \%$ \\
\hline $40 \times 40$ & $1.23 \pm 0.21$ & $1.29 \pm 0.08$ & $1 \%$ & $4 \%$ & $17 \%$ & $7 \%$ \\
\hline $30 \times 30$ & $1.13 \pm 0.43$ & $1.29 \pm 0.18$ & $9 \%$ & $4 \%$ & $34 \%$ & $14 \%$ \\
\hline $20 \times 20$ & $0.91 \pm 0.75$ & $1.24 \pm 0.20$ & $26 \%$ & $0 \%$ & $60 \%$ & $16 \%$ \\
\hline $10 \times 10$ & $0.63 \pm 2.56$ & $0.99 \pm 0.22$ & $49 \%$ & $20 \%$ & $206 \%$ & $18 \%$ \\
\hline
\end{tabular}

literature. Results with the ex vivo sample in Table 4.5 showed that when using the RSLD technique with $20 \lambda \times 20 \lambda$ data blocks the ACS estimation bias (i.e., MPE $=0 \%$ ) and precision (i.e., SDPE $=16 \%$ ) were comparable to the results obtained when using the SLD technique with $40 \lambda \times 40 \lambda$ data blocks $(\mathrm{MPE}=1 \%, \mathrm{SDPE}=17 \%)$. The results with the ex vivo tissue experiment suggest the RSLD technique is applicable beyond numerical and physical phantoms. 

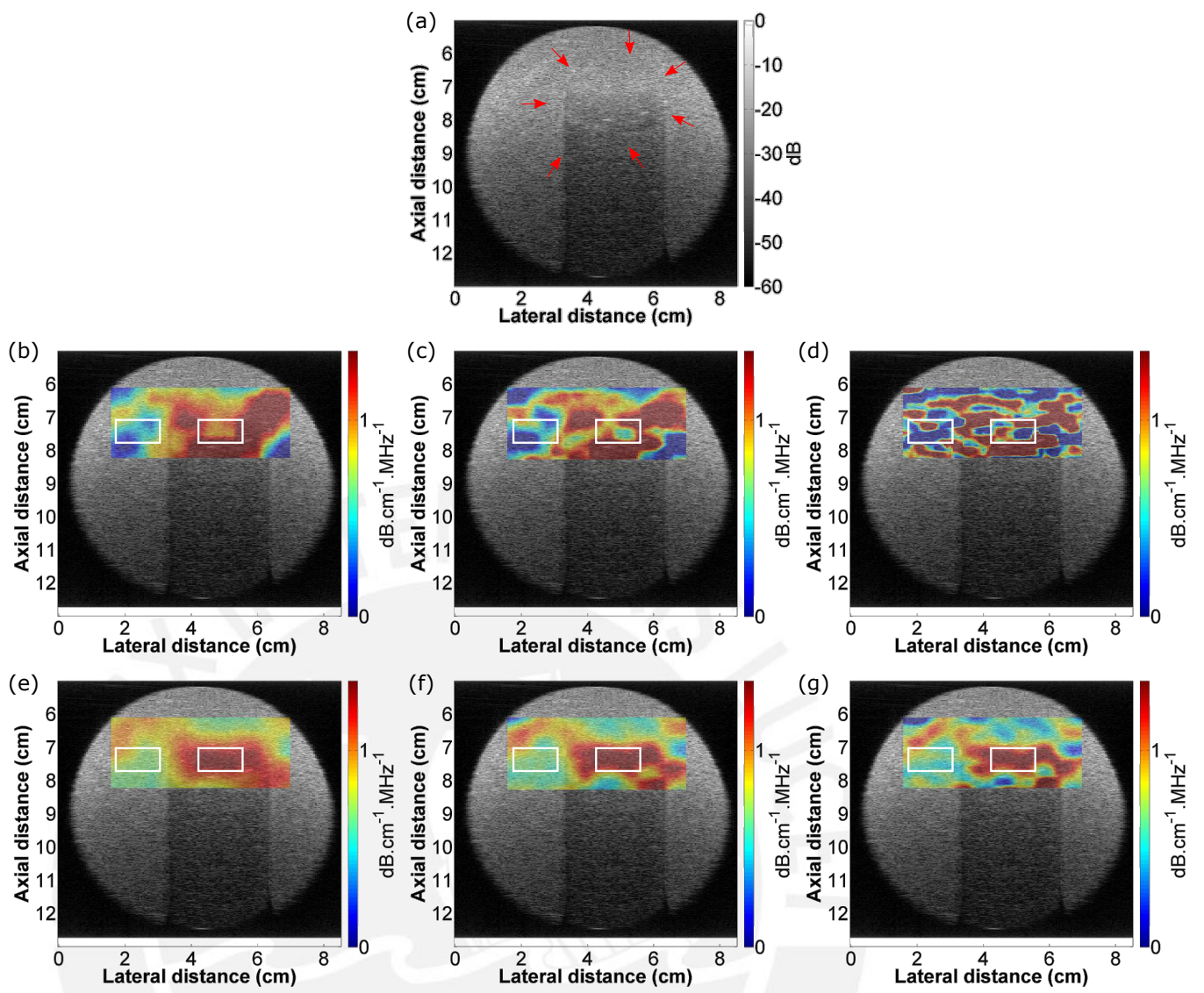

Figure 4.5: B-mode image for ex vivo chicken breast tissue (a) and ACS maps when using the SLD (b)-(d) and RSLD (e)-(g) techniques for data block sizes of $40 \lambda \times 40 \lambda, 30 \lambda \times 30 \lambda$ and $20 \lambda \times 20 \lambda$, respectively. The arrows and solid lines outline the inclusion and regions for the calculation of performance metrics, respectively.

\subsection{In vivo tissue}

Finally, the RSLD technique performance was evaluated with an in vivo breast sample. The ACS maps of the breast tissue sample obtained when using the SLD and the $\operatorname{RSLD}\left(\mu=10^{2.5}\right)$ techniques for data block sizes $30 \lambda \times 30 \lambda, 20 \lambda \times 20 \lambda$ and $10 \lambda \times 10 \lambda$ are shown in Fig. 4.6. Table 4.6 presents the mean and standard deviation of estimated ACS values for $20 \lambda \times 20 \lambda$ regions within the tumor and normal tissue. The MPE and SDPE were not computed for this case because ground truth values were not available.

In [2] ACS values for normal (fat and fibroglandular) tissues and both benign (fibroadenoma) and malignant (carcinoma) tumors in breast estimated in vivo using ultrasonic tomography were reported. These results were mined using the DataThief III software. The ACS of fibroadenoma tumors, fat and fibroglandular tissues in breast were found to be $1.71 \pm 0.49 \mathrm{~dB} \cdot \mathrm{cm}^{-1} \cdot \mathrm{MHz}^{-1}, 0.80 \pm 0.79$ $\mathrm{dB} . \mathrm{cm}^{-1} \cdot \mathrm{MHz}^{-1}$ and $1.00 \pm 0.70 \mathrm{~dB} \cdot \mathrm{cm}^{-1} \cdot \mathrm{MHz}^{-1}$, respectively. These values were in very good agreement with ACS estimates using RSLD both for the fibroadenoma $\left(1.80 \mathrm{~dB} \cdot \mathrm{cm}^{-1} \cdot \mathrm{MHz}^{-1}\right.$ considering the average of values in Table 4.6) and normal surrounding tissues $\left(0.88 \mathrm{~dB} \cdot \mathrm{cm}^{-1} \cdot \mathrm{MHz}^{-1}\right.$ considering the average of values in Table 4.6). As in previous cases, the RSLD technique improved the trade-off between data block size and ACS estimation precision. Moreover, this result suggests that RSLD can 

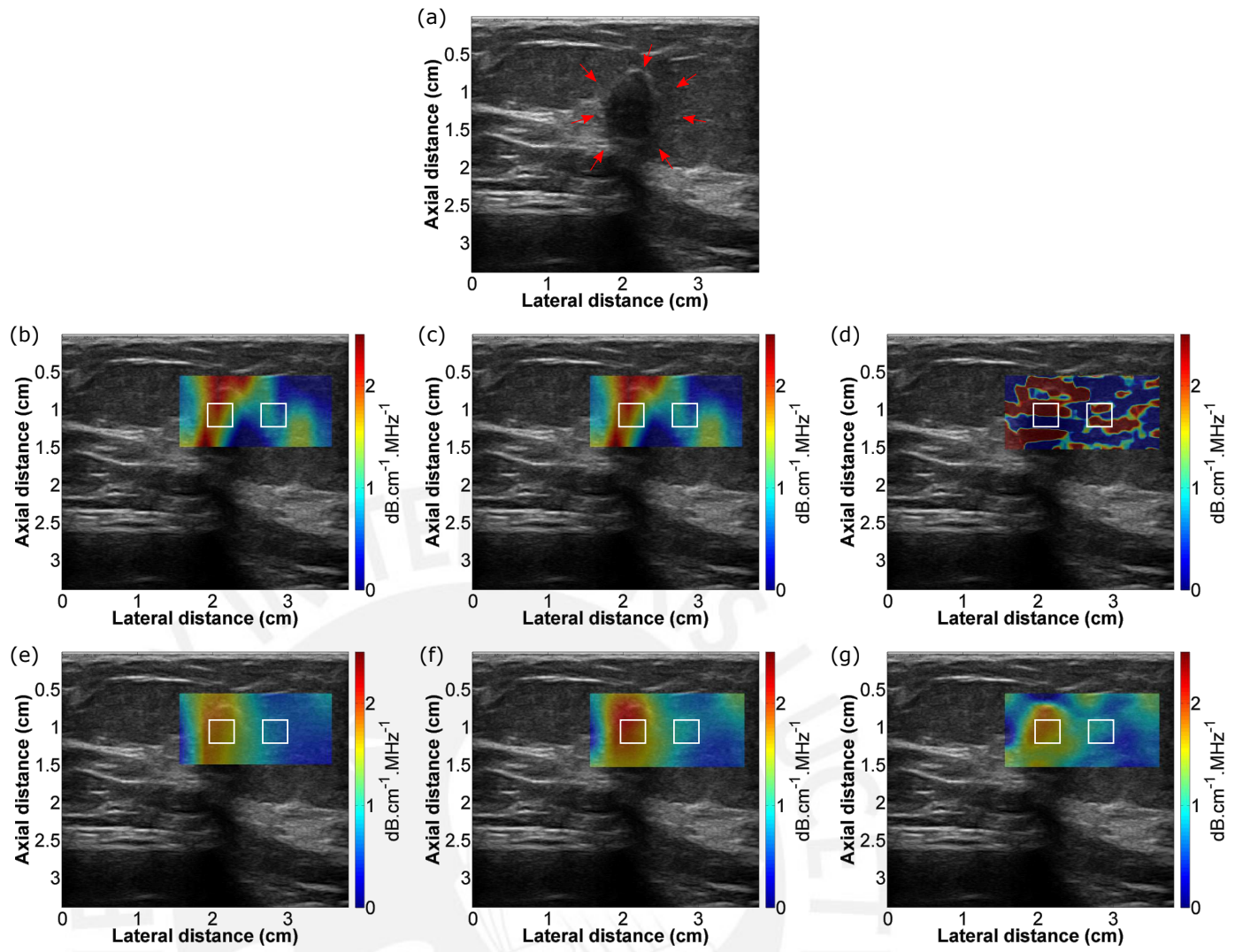

Figure 4.6: B-mode image for in vivo breast tissue (a) and ACS maps when using the SLD (b)-(d) and RSLD (e)-(g) techniques for data block sizes of $30 \lambda \times 30 \lambda, 20 \lambda \times 20 \lambda$ and $10 \lambda \times 10 \lambda$, respectively. The arrows and solid lines outline the tumor and regions for the calculation of performance metrics, respectively.

Table 4.6: Estimated ACS mean and standard deviation of tumor and normal tissue for in vivo tissue obtained when using the SLD and the RSLD techniques.

\begin{tabular}{|c|c|c|c|}
\hline \multirow{2}{*}{ Breast } & $\begin{array}{c}\text { Data block } \\
\text { size }(\lambda)\end{array}$ & \multicolumn{2}{|c|}{ Mean \pm SD $\left(\mathrm{db}^{-\mathrm{cm}^{-1}} \cdot \mathrm{MHz}^{-1}\right)$} \\
\cline { 2 - 4 } & SLD & RSLD \\
\hline \hline \multirow{3}{*}{ Tumor } & $30 \times 30$ & $1.76 \pm 0.32$ & $1.73 \pm 0.11$ \\
\cline { 2 - 4 } & $20 \times 20$ & $2.29 \pm 0.96$ & $1.97 \pm 0.17$ \\
\cline { 2 - 4 } & $10 \times 10$ & $1.26 \pm 3.48$ & $1.71 \pm 0.11$ \\
\hline \multirow{2}{*}{$\begin{array}{c}\text { Normal } \\
\text { tissue }\end{array}$} & $30 \times 30$ & $0.82 \pm 0.21$ & $0.78 \pm 0.13$ \\
\cline { 2 - 4 } & $20 \times 20$ & $0.81 \pm 0.44$ & $0.95 \pm 0.12$ \\
\cline { 2 - 4 } & $10 \times 10$ & $0.61 \pm 2.73$ & $0.9 \pm 0.14$ \\
\hline
\end{tabular}

be readily used for in vivo imaging, although further studies are required for validating its usefulness in clinical applications. 


\subsection{Contrast-to-noise ratio}

The improvements in visualization of inclusions when using the RSLD method was quantified using the CNR for all inhomogeneous media imaging cases. Figure 4.7 shows the CNR of the SLD (a), $\operatorname{RSLD}(b)$ and gain of the RSLD $\left(\mu=10^{2.5}\right)$ with respect to the SLD technique (c) for the ACS maps from Simulation \#2, the physical phantoms, and ex vivo and in vivo tissues. The values of CNR when using the RSLD were always higher than the CNR values when using the SLD, except for the ex vivo tissue sample at data block sizes of $50 \lambda \times 50 \lambda$ and $40 \lambda \times 40 \lambda$. Typically, gains in CNR were higher for smaller data blocks. For example, the median gains in CNR were 30.7 and 8.3 when using $10 \lambda \times 10 \lambda$ and $20 \lambda \times 20 \lambda$ data blocks, respectively.

(a)

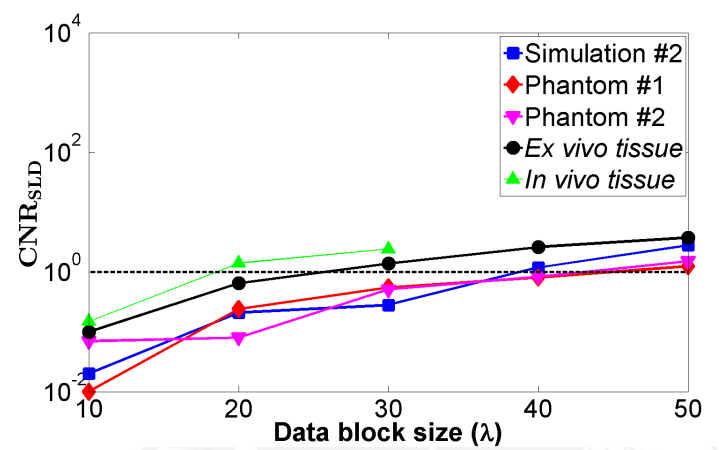

(b)

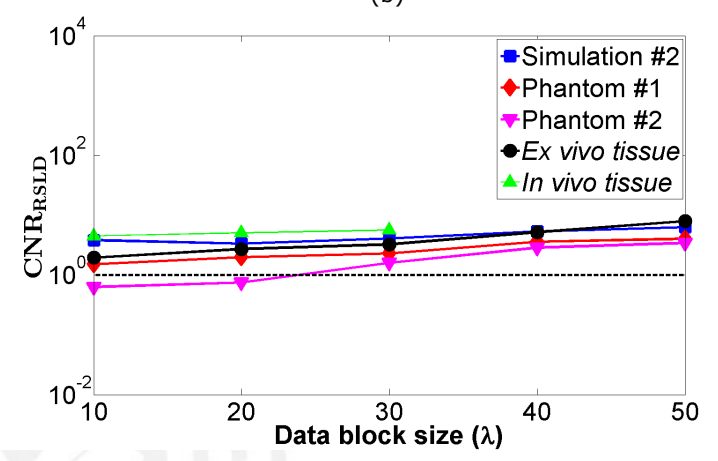

(c)

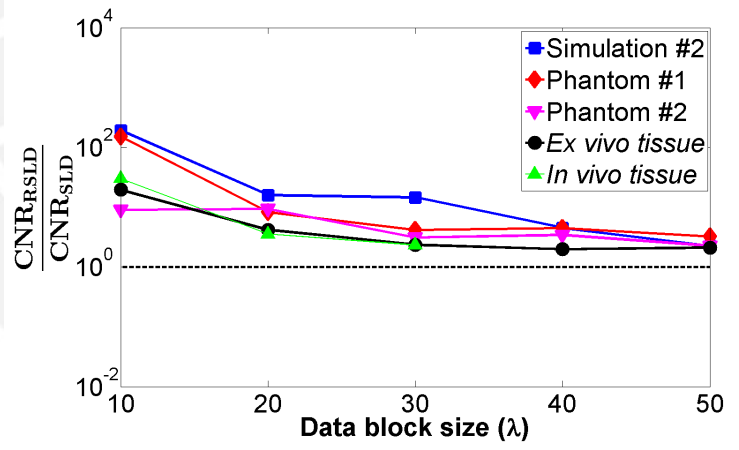

Figure 4.7: CNR of the SLD (a), RSLD (b) and the gain in CNR of the RSLD with respect to the SLD technique (c) of ACS maps for different data block sizes.

It can be observed that for both Simulation \#2 and experiments with the physical phantom, the use of RSLD provided improvements of one and two orders of magnitude in CNR when compared to the use of SLD for data block sizes of $20 \lambda \times 20 \lambda$ and $10 \lambda \times 10 \lambda$, respectively. Gains in CNR were also observed when imaging the ex vivo and in vivo tissues, although a relatively more modest CNR gain was observed (i.e., an order of magnitude when using a data block size of $10 \lambda \times 10 \lambda$ ). However, the trends consistently demonstrated that RSLD provides advantages when imaging inhomogeneous media. 


\subsection{Impact of the regularization parameter value}

As with any regularized inversion method, a significant issue is the proper choice of regularization parameter. In this study, all reconstructions using the RSLD technique were obtained using $\mu=10^{2.5}$. The impact of the choice of regularization parameter was briefly explored using the inhomogeneous physical phantom data set described in Section 3.1.2. The mean, standard deviation and MPE of both inclusion and background regions, as well as the CNR, were calculated when using the RSLD technique for different $\mu$ values and a fixed data block size of $30 \lambda \times 30 \lambda$. The results are shown in Fig. 4.8. It can be observed that for $\mu<10^{1.25}$ no significant improvements in standard deviation reduction or CNR were observed. For $10^{1.25}<\mu<10^{3.75}$ the CNR and standard deviation of ACS maps were improved while keeping the estimation bias below 20\%. Finally, for $\mu>10^{3.75}$ the CNR started to decrease and approach zero, which is an expected result as the total variation term in 2.14 will strongly penalize the spatial variations in ACS, therefore merging the background and inclusion in the reconstructed ACS maps. These results suggest that gains in image quality can be obtained for reasonably large ranges of $\mu$, but the use of techniques for adaptively choosing the regularization parameter should be studied.

(a)

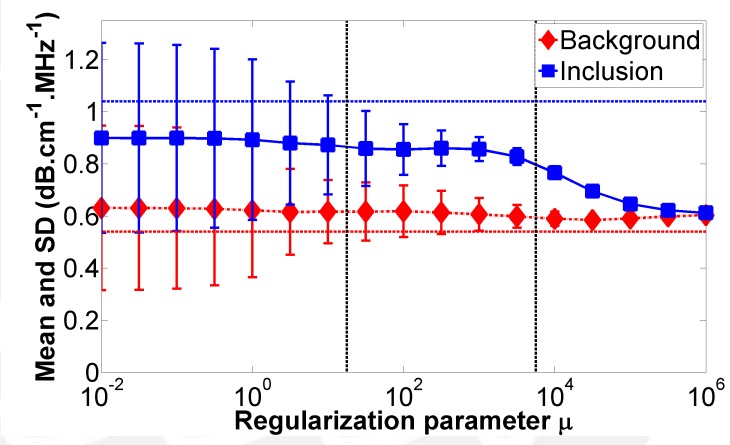

(b)

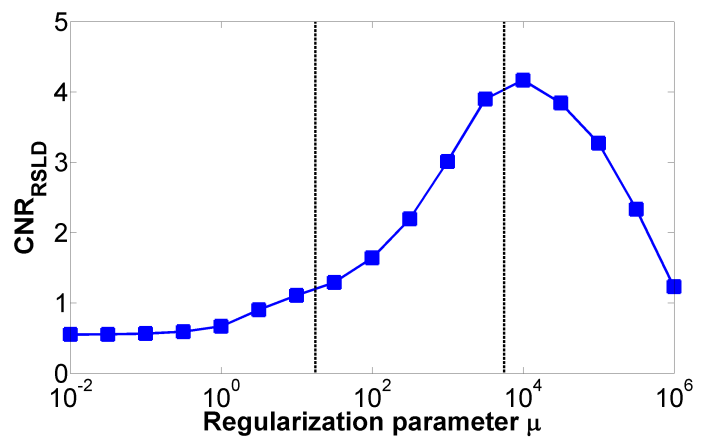

(c)

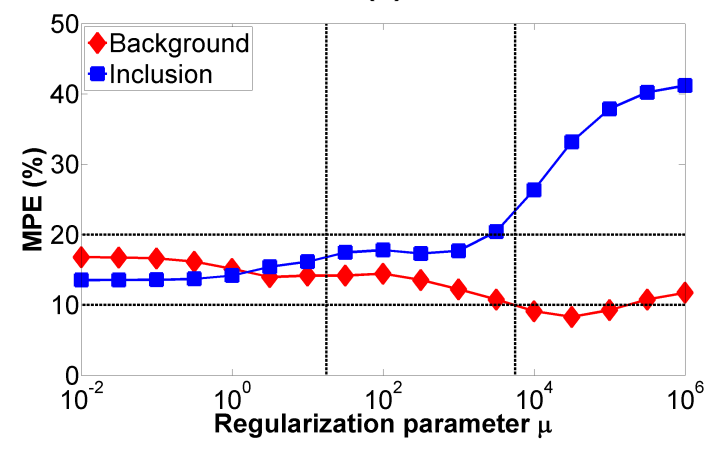

Figure 4.8: Effects of the regularization parameter in the reconstruction of ACS maps using the Phantom \#1. (a) Mean and standard deviation of the inclusion (square markers) and background (diamond markers). The horizontal lines indicate the ground truth values for each region. (b) CNR. (c) MPE of the inclusion (square markers) and background (diamond markers). The horizontal dashed lines mark $10 \%$ and $20 \%$ bias levels. 


\section{Chapter 5}

\section{Conclusion}

Throughout this work, the regularized spectral log difference technique for ultrasonic attenuation imaging has been evaluated using numerical phantoms produced with the k-Wave toolbox and with several types of experimental data including physical phantoms, and ex vivo and in vivo tissues.

The results presented in this work suggest that the regularized spectral log difference technique significantly extends the trade-off between estimation precision and data block size when constructing images of attenuation coefficient slope. Data blocks as small as $20 \lambda \times 20 \lambda$ were shown to provide both accurate and precise attenuation estimates in simulations and experiments with physical phantoms and ex vivo and in vivo tissues, therefore outperforming currently available methods for attenuation coefficient estimation using backscattered ultrasound data. 


\section{Bibliography}

[1] T. L. Szabo, Diagnostic ultrasound imaging: inside out, Academic Press, 1st edition, 2004.

[2] J. Mamou and M. L. Oelze, Quantitative ultrasound in soft tissues, Springer, New York, NY, 2013.

[3] L. E. Kinsler, A. R. Frey, A. B. Coppens, and J. V. Sanders, Fundamentals of acoustics, John Wiley \& Sons, 4th edition, 1999.

[4] N. B. Smith and A. Webb, Introduction to medical imaging: physics, engineering, and clinical applications, Cambridge University Press, 2011.

[5] R. Kuc and M. Schwartz, "Estimating the acoustic attenuation coefficient slope for liver from reflected ultrasound signals," IEEE Transactions on Sonics and Ultrasonics, vol. 26, no. 5, pp. 353-361, 1979.

[6] R. Kuc, "Clinical application of an ultrasound attenuation coefficient estimation technique for liver pathology characterization," IEEE Transactions on Biomedical Engineering, vol. 27, no. 6, pp. 312-319, 1980.

[7] N. F. Maklad, Ophir J., and V. Balsara, "Attenuation of ultrasound in normal liver and diffuse liver disease in vivo," Ultrasonic Imaging, vol. 6, no. 2, pp. 117-125, 1984.

[8] Y. Fujii, N. Tanguchi, K. Itoh, K. Shigeta, Y. Wang, J. W. Tsao, K. Kumasaki, and T. Itoh, "A new method for attenuation coefficient measurement in the liver," Journal of Ultrasound in Medicine, vol. 21, no. 7, pp. 783-788, 2002.

[9] Y. Kanayama, N. Kamiyama, K. Maruyama, and Y. Sumino, "Real-time ultrasound attenuation imaging of diffuse fatty liver disease," Ultrasound in Medicine and Biology, vol. 39, no. 4, pp. 692-705, 2013.

[10] Y. Fujii, N. Taniguchi, K. Itoh, and K. Omoto, "Attenuation coefficient measurement in the thyroid," Journal of Ultrasound in Medicine, vol. 22, no. 10, pp. 1067-1073, 2003.

[11] J. Rouyer, T. Cueva, T. Yamamoto, A. Portal, and R. Lavarello, "In vivo estimation of attenuation and backscatter coefficients from human thyroids," IEEE Transactions on Ultrasonics, Ferroelectrics, and Frequency Control, vol. 63, no. 9, pp. 1253-1261, 2016.

[12] F. T. D'Astous and F. S. Foster, "Frequency dependence of ultrasound attenuation and backscatter in breast tissue," Ultrasound in Medicine and Biology, vol. 12, no. 10, pp. 795-808, 1986. 
[13] C. H. Chang, S. W. Huang, H. C. Yang, Y. H. Chou, and P. C. Li, "Reconstruction of ultrasonic sound velocity and attenuation coefficient using linear arrays: Clinical assessment," Ultrasound in Medicine and Biology, vol. 33, no. 11, pp. 1681-1687, 2007.

[14] K. Nam, J. A. Zagzebski, and T. J. Hall, "Quantitative Assessment of in vivo Breast Masses using Ultrasound Attenuation and Backscatter," Ultrasonic Imaging, vol. 35, no. 2, pp. 146-161, 2013.

[15] L. S. Wilson, D. E. Robinson, K. A. Griffiths, A. Manoharan, and B. D. Doust, "Evaluation of ultrasonic attenuation in diffuse diseases of spleen and liver," Ultrasonic Imaging, vol. 9, no. 4, pp. 236-247, 1987.

[16] J. Ophir, N. F. Maklad, and R. H. Bigelow, "Ultrasonic attenuation measurements of in vivo human muscle," Ultrasonic Imaging, vol. 4, no. 3, pp. 290-295, 1982.

[17] B. I. Raju and M. A. Srinivasan, "High-frequency ultrasonic attenuation and backscatter coefficients of in vivo normal human dermis and subcutaneous fat," Ultrasound in Medicine and Biology, vol. 27, no. 11, pp. 1543-1556, 2001.

[18] H. Piotrzkowska-Wroblewska, J. Litniewski, E. Szymanska, and A. Nowicki, "Quantitative sonography of basal cell carcinoma," Ultrasound in Medicine and Biology, vol. 41, no. 3, pp. 748-759, 2015.

[19] M. Z. Kiss, T. Varghese, and M. A. Kliewer, "Ex vivo ultrasound attenuation coefficient for human cervical and uterine tissue from 5 to $10 \mathrm{MHz}$," Ultrasonics, vol. 51, no. 4, pp. 467-471, 2011.

[20] B. L. McFarlin, V. Kumar, T. A. Bigelow, D. G. Simpson, R. C. White-Traut, J. S. Abramowicz, and W. D. O'Brien, Jr., "Beyond cervical length: A pilot study of ultrasonic attenuation for early detection of preterm birth risk," Ultrasound in Medicine and Biology, vol. 41, no. 11, pp. 3023 3029, 2015.

[21] H. Shi, H. Tu, R. J. Dempsey, and T. Varghese, "Ultrasonic attenuation estimation in small plaque samples using a power difference method," Ultrasonic Imaging, vol. 29, no. 1, pp. 15-30, 2007.

[22] M. L. Oelze and W. D. O’Brien, Jr., "Frequency-dependent attenuation-compensation functions for ultrasonic signals backscattered from random media," The Journal of the Acoustical Society of America, vol. 111, no. 5, pp. 2308-2319, 2002.

[23] T. A. Bigelow, "Estimating the total ultrasound attenuation along the propagation path by applying multiple filters to backscattered echoes from a single spherically focused source," 2010.

[24] K. Nam, J. A. Zagzebski, and T. J. Hall, "Simultaneous backscatter and attenuation estimation using a least squares method with constraints," Ultrasound in Medicine and Biology, vol. 37, no. 12, pp. 2096-2104, 2011.

[25] A. D. Pawlicki and W. D. O'Brien, Jr., "Method for estimating total attenuation from a spatial map of attenuation slope for quantitative ultrasound imaging," Ultrasonic Imaging, vol. 35, no. 2, pp. 162-172, 2013.

[26] K. Samimi and T. Varghese, "Performance evaluation of the spectral centroid downshift method for attenuation estimation," IEEE Transactions on Ultrasonics, Ferroelectrics, and Frequency Control, vol. 62, no. 5, pp. 871-880, 2015. 
[27] Y. Labyed and T. A. Bigelow, "A theoretical comparison of attenuation measurement techniques from backscattered ultrasound echoes," The Journal of the Acoustical Society of America, vol. 129, no. 4, pp. 2316-2324, 2011.

[28] H. Kim and T. Varghese, "Hybrid spectral domain method for attenuation slope estimation," Ultrasound in Medicine and Biology, vol. 34, no. 11, pp. 1808-1819, 2008.

[29] K. Samimi and T. Varghese, "Lower bound on estimation variance of the ultrasonic attenuation coefficient using the spectral-difference reference phantom method," Ultrasonic Imaging, pp. $1-21,2016$.

[30] K. Samimi and T. Varghese, "Optimum diffraction-corrected frequency-shift estimator of the ultrasonic attenuation coefficient," IEEE Transactions on Ultrasonics, Ferroelectrics, and Frequency Control, vol. 63, no. 5, pp. 691-702, 2016.

[31] N. Rubert and T. Varghese, "Scatterer number density considerations in reference phantom-based attenuation estimation," Ultrasound in Medicine and Biology, vol. 40, no. 7, pp. 1680-1696, 2014.

[32] H. Tu, T. Varghese, E. L. Madsen, Q. Chen, and J. A. Zagzebski, "Ultrasound attenuation imaging using compound acquisition and processing," Ultrasonic Imaging, vol. 25, no. 4, pp. 245-261, 2003.

[33] O. Zenteno, A. Luchies, M. Oelze, and R. Lavarello, "Improving the quality of attenuation imaging using full angular spatial compounding," in Proceedings of the IEEE International Ultrasonics Symposium, 2014, pp. 2426-2429.

[34] G. Ghoshal and M. L. Oelze, "Time domain attenuation estimation method from ultrasonic backscattered signals," The Journal of the Acoustical Society of America, vol. 132, no. 1, pp. 533-543, 2012.

[35] N. Ilyina, J. Hermans, E. Verboven, K. Van Den Abeele, E. D’Agostino, and J. D'hooge, “Iterative reconstruction of the ultrasound attenuation coefficient from the backscattered radio-frequency signal," in Proceedings of the IEEE International Ultrasonics Symposium, 2014, pp. 2406-2409.

[36] A. Coila, J. Rouyer, O. Zenteno, and R. Lavarello, "A regularization approach for ultrasonic attenuation imaging," in Proceedings of the IEEE 13th International Symposium on Biomedical Imaging (ISBI), apr 2016, pp. 469-472.

[37] X. Chen, D. Phillips, K. Q. Schwarz, J. G. Mottley, and K. J. Parker, "The measurement of backscatter coefficient from a broadband pulse-echo system: A new formulation," IEEE Transactions on Ultrasonics, Ferroelectrics, and Frequency Control, vol. 44, no. 2, pp. 515-525, 1997.

[38] O. Zenteno, W. Ridgway, S. Sarwate, M. Oelze, and R. Lavarello, "Ultrasonic attenuation imaging in a rodent thyroid cancer model," in Proceedings of the IEEE International Ultrasonics Symposium, 2013, pp. 88-91.

[39] C. R. Vogel, Computational methods for inverse problems, Society for Industrial and Applied Mathematics, Philadelphia, PA, 2002.

[40] P. Rodríguez, "Total variation regularization algorithms for images corrupted with different noise models: A review," Journal of Electrical and Computer Engineering, vol. 2013, pp. 1-18, 2013. 
[41] P. Getreuer, "Total variation deconvolution using split Bregman," Image Processing On Line, vol. 2, pp. 158-174, 2012.

[42] S. Boyd, N. Parikh, E. Chu, B. Peleato, and J. Eckstein, "Distributed optimization and statistical learning via the alternating direction method of multipliers," Foundations and Trends in Machine Learning, vol. 3, no. 1, pp. 1-122, 2011.

[43] B. E. Treeby, J. Jaros, A. P. Rendell, and B. T. Cox, "Modeling nonlinear ultrasound propagation in heterogeneous media with power law absorption using a k-space pseudospectral method," The Journal of the Acoustical Society of America, vol. 131, no. 6, pp. 4324-4336, 2012.

[44] S. A. Goss, R. L. Johnston, and F. Dunn, "Comprehensive compilation of empirical ultrasonic properties of mammalian tissues," The Journal of the Acoustical Society of America, vol. 64, no. 2, pp. 423-457, 1978.

[45] E. L. Madsen, J. A. Zagzebski, R. A. Banjavie, and R. E. Jutila, "Tissue mimicking materials for ultrasound phantoms," Medical Physics, vol. 5, no. 5, pp. 391-394, 1978.

[46] K. A. Wear, T. A. Stiles, G. R. Frank, E. L. Madsen, F. Cheng, E. J. Feleppa, C. S. Hall, B. S. Kim, P. Lee, W. D. O’Brien Jr., M. L. Oelze, B. I. Raju, K. K. Shung, T. A. Wilson, and J. R. Yuan, "Interlaboratory comparison of ultrasonic backscatter coefficient measurements from 2 to 9 MHz," Journal of Ultrasound in Medicine, vol. 24, no. 9, pp. 1235-1250, 2005.

[47] J. Ormachea, R. Lavarello, S. A. McAleavey, K. J. Parker, and B. Castaneda, "Shear wave speed measurements using crawling wave sonoelastography and single tracking location shear wave elasticity imaging for tissue characterization," IEEE Transactions on Ultrasonics, Ferroelectrics, and Frequency Control, vol. 63, no. 9, pp. 1351-1360, 2016.

[48] M. O’Donnell and S. D. Silverstein, “Optimum displacement for compound image generation in medical ultrasound," IEEE Transactions on Ultrasonics, Ferroelectrics, and Frequency Control, vol. 35, no. 4, pp. 470-476, 1988.

[49] B. Hete and K. K. Shung, "Scattering of ultrasound from skeletal muscle tissue," IEEE Transactions on Ultrasonics, Ferroelectrics, and Frequency Control, vol. 40, no. 4, pp. 354-365, 1993. 